\title{
Le marché de Sabra à Beyrouth par l'image et le son. Retour sur une enquête intensive
}

Sabra Market in Beirut through Pictures, Videos and Sounds: Methodological Perspectives

El mercado de Sabra de Beirut a través de la imagen y el sonido. Resultados de una encuesta en profundidad

Houda Kassatly, Nicolas Puig et Michel Tabet

\section{OpenEdition}

\section{Journals}

Édition électronique

URL : https://journals.openedition.org/remi/8179

DOI : $10.4000 /$ remi.8179

ISSN : $1777-5418$

Éditeur

Université de Poitiers

Édition imprimée

Date de publication : 1 décembre 2016

Pagination : $37-68$

ISBN : 979-10-90426-29-0

ISSN : 0765-0752

\section{Référence électronique}

Houda Kassatly, Nicolas Puig et Michel Tabet, « Le marché de Sabra à Beyrouth par l'image et le son. Retour sur une enquête intensive », Revue européenne des migrations internationales [En ligne], vol. 32 n³ et 4 | 2016, mis en ligne le 01 décembre 2018, consulté le 15 avril 2022. URL : http:// journals.openedition.org/remi/8179; DOI : https://doi.org/10.4000/remi.8179 


\section{Le marché de Sabra à Beyrouth par I'image et le son. Retour sur une enquête intensive}

\section{Houda Kassatly ${ }^{1}$, Nicolas Puig ${ }^{2}$ et Michel Tabet ${ }^{3}$}

La présence importante et pérenne de migrants étrangers dans les villes, notamment sur le pourtour méditerranéen, provoque une multiplication des espaces de rencontres entre des populations d'origines différentes et une multiplication de lieux de centralités migrantes ou minoritaires (Raulin : 2009). De sorte que, depuis un moment déjà, la description des expériences de la coprésence devient l'une des entrées de l'étude des migrations internationales (Hily et Berthomière, 2006). On constate ainsi l'émergence de travaux portant sur l'insertion urbaine de groupes migrants et/ou minoritaires en différents lieux tels que le quartier d'Omonia à Athènes (Noussa et Lyons, 2009), Barbès et la Goutte d'or à Paris (Lallemand, 2010 ; Millot, 2013), ou encore la place marchande de Vintimille à la frontière italo-française où cosmopolitisme et ethnicité sont mis en tension (Hily et Rinaudo, 2003).

Le marché de Sabra au Liban présente un caractère multi-ethnique comme il constitue un lieu de regroupement des migrants asiatiques. Nous avons déployé sur ce site différents outils audiovisuels que nous présentons dans ce texte conçu comme une contribution méthodologique aux études sur les interactions dans les situations de coprésence ${ }^{4}$.

\footnotetext{
1 Chercheuse associée à I'UIR MÉMOIRE, Université Saint-Joseph/CEMAM, Bât. C, rue de Damas, Beyrouth, B.P. 17-5208 Mar Mikhaël Beyrouth, 1104 2020, Liban ; hodak@ arcenciel.org

2 Anthropologue, Chargé de recherche, URMIS, Université Paris-Diderot/IRD, Case 7027, 75205 Paris Cedex 13 ; nicolas.puig@ird.fr

3 Membre associé au Laboratoire d'Anthropologie Sociale, 52 rue du Cardinal Lemoine, 75005 Paris ; michel@zwyx.org

4 Ces travaux ont bénéficié du soutien du programme CEDRE du CNRS libanais et du MAE français, projet "Modes de présence des migrants au Liban ", coordonné par Liliane Kfoury, UIR MÉMOIRE (CEMAM, Université Saint-Joseph) et Nicolas Puig, URMIS (2013-2014), ainsi que d'une aide du CNRS libanais en 2012 dans la cadre d'une recherche portant sur les travailleurs étrangers associant I'Institut français du Proche-Orient (IFPO), I'Institute for Migration Studies (IMS) de la Lebanese American University et le Centre d'études sur le monde arabe moderne (CEMAM) de I'Université Saint-Joseph.
} 
Cet important marché regroupe des bouchers et des vendeurs de fruits et légumes palestiniens, libanais et syriens. Or, depuis 2011, tous les dimanches, jour de forte affluence, des Bangladais, arrivés en nombre au Liban depuis quelques années - ils seraient à présent plus de 40000 selon une estimation de l'ambassade du Bangladesh -, investissent ce marché avec leurs propres commerces constitués de légumes asiatiques, poissons de rivière, épices, produits de beauté, DVD, etc. Ils installent leurs étals de fortune devant les échoppes des bouchers, à côté des charrettes à bras des locaux, dans les interstices du marché, créant des cohabitations inédites dans un lieu saturé, autant par la présence humaine que par les significations hétérogènes que chacun $y$ dépose. Depuis l'été 2014, les autorités tentent de circonscrire les activités des Bangladais dans une zone autour du carrefour de Sabra, mais les tensions et tractations sur les emplacements n'en ont pas diminué pour autant. Elles se sont même accrues du fait du succès du marché bangladais. L'action à visée régulatrice de la gendarmerie (darak) de Beyrouth concerne d'ailleurs tout autant les marchands locaux quasiment tous dépourvus de patente ainsi que les Syriens récemment arrivés qui déploient de petits commerces le long de l'avenue de Sabra.

Nous avons formé une petite équipe spécialisée en anthropologie visuelle et sonore, Houda Kassatly (photographies), Michel Tabet (vidéos) et Nicolas Puig (enregistrements sonores). Nous avions pour objectif d'approcher l'expérience sensorielle et relationnelle des acteurs du marché de Sabra à partir de séquences de diverses natures. Nous cherchions à dégager les propriétés visuelles et sonores du lieu et à en relater les petits évènements sociaux dont les enchaînements instaurent un ordre social qui lui est spécifique.

Ce travail procède d'une enquête intensive. Elle consiste en l'investissement collectif d'un terrain circonscrit pour effectuer des observations sur des temps précis en déployant des outils d'observation variés. Dans le cas d'espèce, il $s^{\prime}$ agit donc du marché de Sabra au moment où des migrants du Bangladesh s'y installent tous les dimanches matins, dès avant l'aube, jusqu'au début de l'aprèsmidi. L'enquête est discontinue, elle s'appuie sur les rythmes du lieu pour sa réalisation. Elle se distingue de ce point de vue des terrains extensifs : I'investissement d'un espace social par un chercheur sur le temps long plus ou moins continu, jusqu'au "terrain résident ", classique de l'anthropologie, où le chercheur s'établit parmi les personnes appartenant aux milieux et groupes qu'il étudie.

Néanmoins, le déploiement des outils audiovisuels n'aurait pas été possible sans l'établissement d'une relation privilégiée avec des occupants du marché qui furent pour nous à la fois des guides et des médiateurs auprès de leurs groupes respectifs. Le vendeur de bananes syrien Abu-I-Izz nous a présentés aux marchands voisins, palestiniens pour la plupart, et son stand constituait une base stabilisée d'observation, et Hassan, originaire du Bangladesh, nous a introduits auprès des membres de sa famille et de certains de ses amis. Nous avons été, à des degrés différents d'implication, en interaction avec les habitués du marché et sommes devenus pour eux des figures familières au fil d'une enquête entreprise en novembre 2012, poursuivie régulièrement durant l'année qui a suivi et complétée depuis 2014 par des observations régulières permettant d'actualiser les données recueillies et de suivre l'évolution du marché et de ses acteurs. Nous nous sommes présentés comme des chercheurs faisant une enquête sur 
le marché dans le cadre d'un travail universitaire. Notre assiduité a permis de créer une familiarité, d'instaurer avec certaines personnes des relations suivies.

Dans cet article, nous décrivons les dispositifs méthodologiques déployés : la photographie, la vidéo et le son, leur réception par les acteurs et les effets de connaissance attendus. Chaque membre de l'équipe relate son approche du terrain, la façon dont il I'a investi et livre quelques résultats de recherche. En amont, l'ajustement des attitudes face à un appareil photo, les effets de pro-filmie ou les réactions à l'écoute des sons du marché constituent autant de pistes précieuses pour comprendre les perceptions des acteurs du marché. Puis I'approche audiovisuelle délivre des informations sur les façons d'apparaître, de se rencontrer et d'échanger des groupes d'origines et de statuts différents qui se côtoient sur le marché.

Le dispositif déployé à Sabra a permis de recueillir un matériel particulièrement conséquent constitué de photographies, vidéos et enregistrements sonores. La diffusion ${ }^{5}$ de ce matériel n'est pas sans soulever des questions quant au droit à l'image, et au son - paroles échangées sur le marché transparaissant des captations sonores -, questionnement que renforce le caractère vulnérable des personnes présentes, notamment des migrants. Depuis Bateson et les 25000 clichés qu'il a pris à Bali, la question du droit à l'image, de la restitution ou encore de l'anonymat s'est, en effet, imposée dans la réflexion sur les bonnes pratiques de la recherche audiovisuelle. Faut-il par exemple obtenir une autorisation explicite de publication d'une photographie prise sur le marché, sachant que le corpus atteint près de 500 photos, au risque de rendre le matériel de l'enquête impossible à publier et de compromettre ainsi la restitution d'un travail dont le motif public réside dans une forme de soutien aux migrants ${ }^{6}$ ? Est-elle même " compatible avec les enjeux d'une recherche scientifique?" (Jarrigeon, 2015 : 226). Nous apporterons des questions pragmatiques à ces questions sérieuses, non sans prendre rendez-vous pour une discussion future sur cette question de l'éthique visuelle, à laquelle il faudrait ajouter une " éthique sonore ", que repose de façon spécifique l'enquête de Sabra.

Nous préciserons donc au cas par cas les conduites adoptées, les accords échangés, explicites ou implicites, et les précautions prises lors des captations visuelles et sonores - la relation d'enquête entre les chercheurs et les acteurs étant elle-même une source de connaissance, en plus d'être une préoccupation éthique jusqu'aux modalités de diffusion du matériel dans le cadre de cet article et au-delà.

5 La photographie dans les espaces publics en milieu urbain ne fait pas l'objet d'interdit particulier tant qu'elle ne prend pas un caractère trop intrusif du moins du point de vue législatif (au moins en France et en Suisse, il faudrait voir ce qu'il en est au Liban), elle demeure bien entendu redevable de l'éthique de recherche. II n'en reste pas moins qu'en conséquence, " la préoccupation principale pour la sociologie [et l'anthropologie] visuelle se situe dans doute moins au niveau de la prise de vue qu'à celui de la publication et de la diffusion des images " (Meyer, $2010: 161$ ).

6 Par exemple sous forme d'exposition, à l'instar de celle organisée en 2014 à l'institut français - ou travaille Hassan comme employé de la société de nettoyage en charge du ménage des locaux - qui a permis une certaine restitution tout en présentant les migrant(e)s sous un jour favorable dans un pays où ils sont victimes de regards racisants et stigmatisants. Une seconde exposition est prévue par laquelle nous souhaitons continuer ce modeste travail de familiarisation du public libanais à la richesse des univers migrants. 


\section{Un moment photographique dans le marché bangla- dais de Sabra : la photographie à l'épreuve de la précarité. Contribution d'Houda Kassatly}

Lorsque j'ai été sollicitée pour observer le souk des Bangladais à Sabra, j'ai tout de suite accepté. Des années de travail sur le terrain alliant photographie et recherche anthropologique $\mathrm{m}^{\prime}$ ont rompu à des pratiques d'observation mixte. Mais cette fois, c'est uniquement en tant que photographe que j'ai souhaité intervenir, préférant me cantonner à l'aspect visuel de la recherche, pragmatiquement pour me sentir plus libre, car la double approche anthropologique et photographique, au-delà de sa complémentarité, induit des démarches différentes souvent laborieuses à mener de front.

Sur ce terrain un peu particulier du carrefour de Sabra, j'étais confrontée à un lieu en mouvement, en composition et recomposition continuelle. Le changement s'inscrit surtout dans l'espace puisque depuis le moment où l'enquête a été initiée, le déplacement géographique du souk est continu : d'un changement radical de rue à quelques mètres en arrière ou en avant, selon les tentatives des migrants pour grappiller quelques centimètres et des propriétaires pour les reprendre, dans le but de réaffirmer avec régularité leur identité de décideurs. Souvent palestiniens, ces propriétaires, eux-mêmes étrangers, se situent sur une échelle d'ancienneté qui hiérarchise les arrivants et qui confère à ceux entrés en premier dans le pays des droits d' " ainesse ".

Les atermoiements constants pour valider la présence migrante sont du fait des locaux, mais aussi des différentes municipalités qui se partagent la gérance des rues sur lesquelles les migrants exercent leurs activités. C'est donc dans ce lieu volatile, mais en même temps consacré, que j'ai évolué durant quelques mois et où je poursuis des incursions dominicales continues bien qu'irrégulières. Dominicales dans un souk à temporalité particulière puisque son ouverture est entièrement tributaire de la disponibilité des migrants, qu'ils soient vendeurs ou clients. Pour exercer cette activité, annexe de leur emploi principal (en entreprise ou à domicile), ou pour profiter de ce souk, les migrants ne peuvent disposer que du dimanche, leur jour de congé, du moins pour ceux qui n'en sont pas privés par des employeurs rétifs à leur accorder ce droit.

Ma présence sur le terrain avait été en quelque sorte balisée par le travail de Nicolas Puig qui avait commencé l'enquête bien avant moi en 2012 et y avait noué de nombreuses relations. Mais comme toujours dans les lieux sensibles, l'apparition d'un appareil photographique trace à elle seule les contours d'un conflit. L'intrusion de ce médium entraîne automatiquement réflexe de suspicion et strates hiérarchisées de méfiance. Mon expérience photographique qui a débuté au cours des années de guerre civile s'inscrit dans une composition constante avec ce que j'ai appris à nommer "l'espionite aiguë des miliciens ". J'étais déjà habituée à côtoyer des personnes pour lesquelles la photographie est synonyme de péril et porte en elle les germes d'un risque certain, quelle que soit l'identité du photographe. Les avancées technologiques, les Google Maps ou autres possibilités de renseignements n'ont pas ôté aux différents belligérants cette méfiance viscérale envers un objet qu'ils continuent à considérer comme une arme dont la dangerosité est, à leurs yeux, avérée. Et cet état de fait se vérifie évidemment dans le souk de Sabra, lieu conflictuel avec sa cohorte de réfugiés anciens ou nouveaux et son lourd passé. 
De plus, à la différence du reportage photographique ordinaire, I'anthropologie visuelle suppose une présence réitérée qui crée une dynamique différente puisqu'elle s'étale dans la durée. Et cette durée peut susciter une montée de la méfiance ou créer un rapport privilégié entre le photographe et les individus présents qu'ils soient témoins ou objets de la photographie. La récurrence et le retour régulier suscitent la crainte avec un présupposé, " s'ils reviennent, c'est que ça cache quelque chose ", qui se manifeste par la question de savoir pourquoi la recherche n'est pas encore achevée.

\section{Photographe/locaux}

La crainte de l'intrusion de la photographie se manifeste chez les locaux (chauffeurs de taxi, locataires ou propriétaires des échoppes fixes ou des charrettes, passants ou clients du souk ordinaire) par des réflexes de protection qui se traduisent par des questionnements répétés sur les raisons de ma présence. Ces divers questionnements s'accompagnent d'un spectre d'attitudes; de la simple curiosité et souhait de clarification à une inquisition en bonne et due forme accompagnée d'agressivité, voire de dictats m'interdisant d'exercer mon activité. Les plus virulents sont portés, une fois encore, par le besoin d'affirmer leur présence et de démontrer qu'ils sont les décideurs, susceptibles d'agréer ou non ma présence.

Le résultat de ce jeu des questions-réponses est divers. Lorsque la réponse est jugée satisfaisante ou rassurante, le lien s'établit, la présence est justifiée, mais plus encore le rapport peut fondamentalement se transformer, même avec ceux qui ont fait montre au départ de la plus grande défiance. Ils peuvent même devenir ceux qui vont cautionner ma présence en se chargeant d'en expliquer les raisons à ceux qui, me voyant pour la première fois, $\mathrm{m}^{\prime}$ interrogent à ce sujet.

La nature d'un lieu passant comme un souk détermine à elle seule la difficulté $d^{\prime} y$ stationner. Clients et visiteurs changent constamment et chaque passage apporte un nouveau lot de personnes tout aussi investigatrices et qui à chaque fois, risquent de remettre en cause la validité de mon travail. Mais la présence de " constants", que ce soit au niveau des " riverains" ou au niveau des migrants (vendeurs, organisateurs du souk, etc.), des chauffeurs de taxi, des commerçants, des propriétaires des boutiques fixes, permet en définitive et en quelque sorte de s'établir dans le lieu.

Lorsque la confiance s'installe et que le photographe est toléré, voire accepté, il se transmue en photographe-confesseur. Grâce à sa dimension de chercheur et de professionnel affilié à une université, sa stature peut en imposer. II devient alors un interlocuteur privilégié, voire une sorte de réceptacle de confession des difficultés quotidiennes, mais surtout des considérations politiquement incorrectes qui s'expriment sans garde-fous, ni barrières. Ces confessions portent essentiellement sur la présence migrante et sur ce qu'elle cause et entraîne au regard de ses interlocuteurs locaux ; chaos, désordre, saleté récurrente. Le photographe est invectivé, il est pris à témoin de la colonisation de l'espace par les migrants et en conséquence de toutes les nuisances dont ils sont la cause. Ceux qui s'adressent à lui peuvent exprimer un racisme sans fard, certains vont jusqu'à comparer les migrants à des animaux. 


\section{Photographe/migrants}

Les migrants dont je suis chargée d'observer visuellement les échanges et les pratiques perçoivent différemment ma présence. Eux aussi posent des questions et attendent des réponses. Le souk de Sabra, c'est l'intrusion de la photographie dans un environnement sensible. L'explication rassure une population fragile et vulnérable. Parmi les migrants, il y a tous les illégaux qui craignent la photographie en tant que document susceptible d'être utilisé contre eux et ne souhaitent pas être photographiés pour des raisons évidentes. Avec eux, le rapport est complexe. II y a d'abord une double peur, celle d'être arrêté (peur que nous pouvons retrouver aussi chez ceux qui sont en règle avec les administrations publiques) et la crainte de se retrouver en infraction par rapport à la loi parce qu'ils exercent une activité à côté de celle pour laquelle ils sont venus. Peu d'entre eux se "permettent " toutefois d'être agressifs ou offensifs. En tant qu'étrangers, non soutenus par des structures, en situation précaire, ils ne s'octroient pas de voix au chapitre et acceptent l'anthropologue-photographe comme faisant partie d'un ordre qui leur échappe en grande partie. C'est manifestement le cas pour les femmes apparaissant dans les clichés 1 et 2 dont les visages ont été floutés en conséquence.

Pourtant, au-delà de ces limitations, des liens se forment. Avec l'habitude et le fait qu'aucune suite négative ne résulte du travail photographique, les migrants ne perçoivent plus le photographe comme source de tracas ou d'ennuis et acquièrent la certitude qu'il ne représente aucune autorité susceptible de vouloir leur causer un tort quelconque.

Je pouvais également compter sur le support du ou des migrants, qui sont mis au fait des détails de l'enquête et des raisons de la recherche. Ce sont alors les " amis " qui se partagent la responsabilité d'être aux premières loges de l'information et, de ce fait, prennent à leur charge la tâche de rassurer leurs compatriotes (Photo 3).

L'intérêt du photographe pour leurs marchandises et leurs recettes participe également de la réduction des tensions. Les migrants répondent avec facilité à ces inquisitions culinaires qui permettent d'amorcer le dialogue (Photos 4 à 7).

Photos 1 et 2 : Femmes migrantes à Sabra
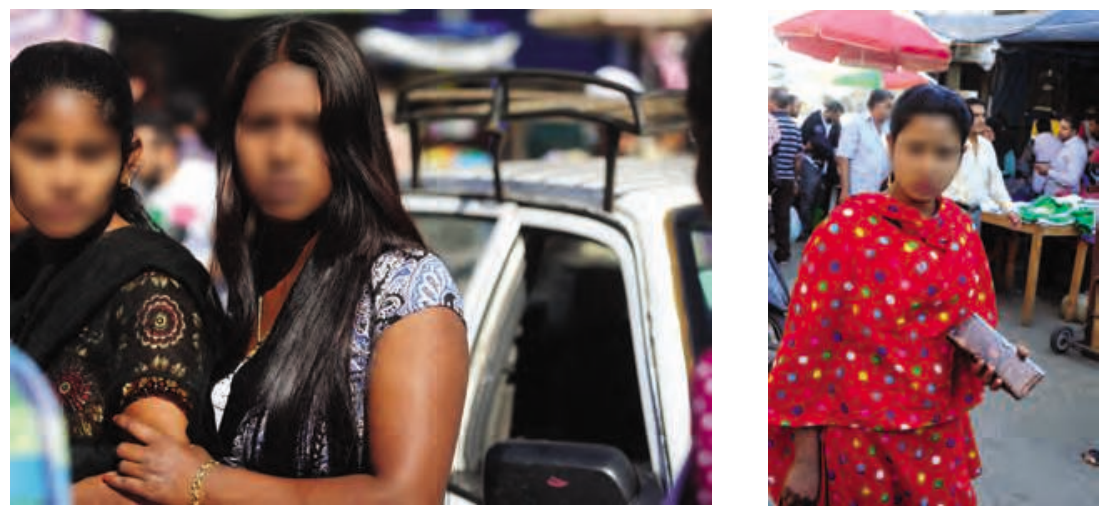

Crédit : H. Kassatly, Marché de Sabra, Beyrouth, 2012 et 2013. 
Photo 3 : L'informateur ami

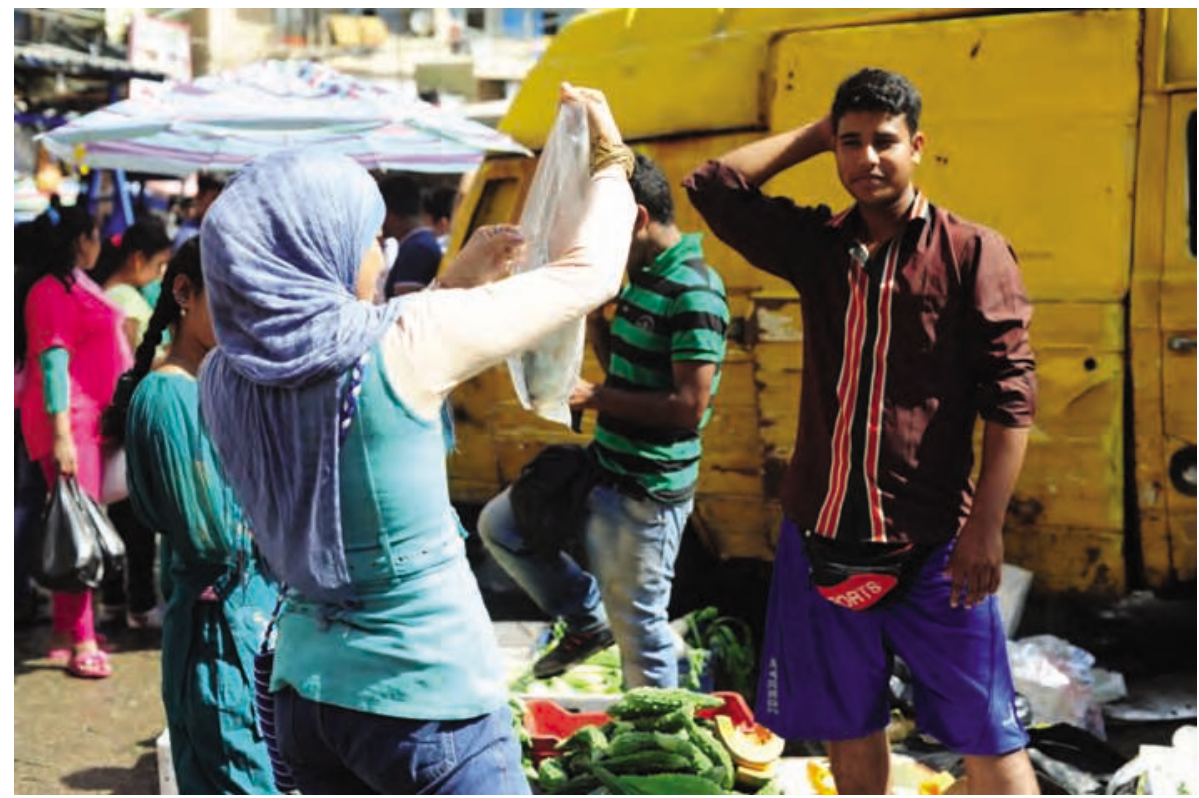

Crédit : H. Kassatly, Marché de Sabra, Beyrouth, 2013.

Photos 4, 5, 6 et 7 : Légumes du souk

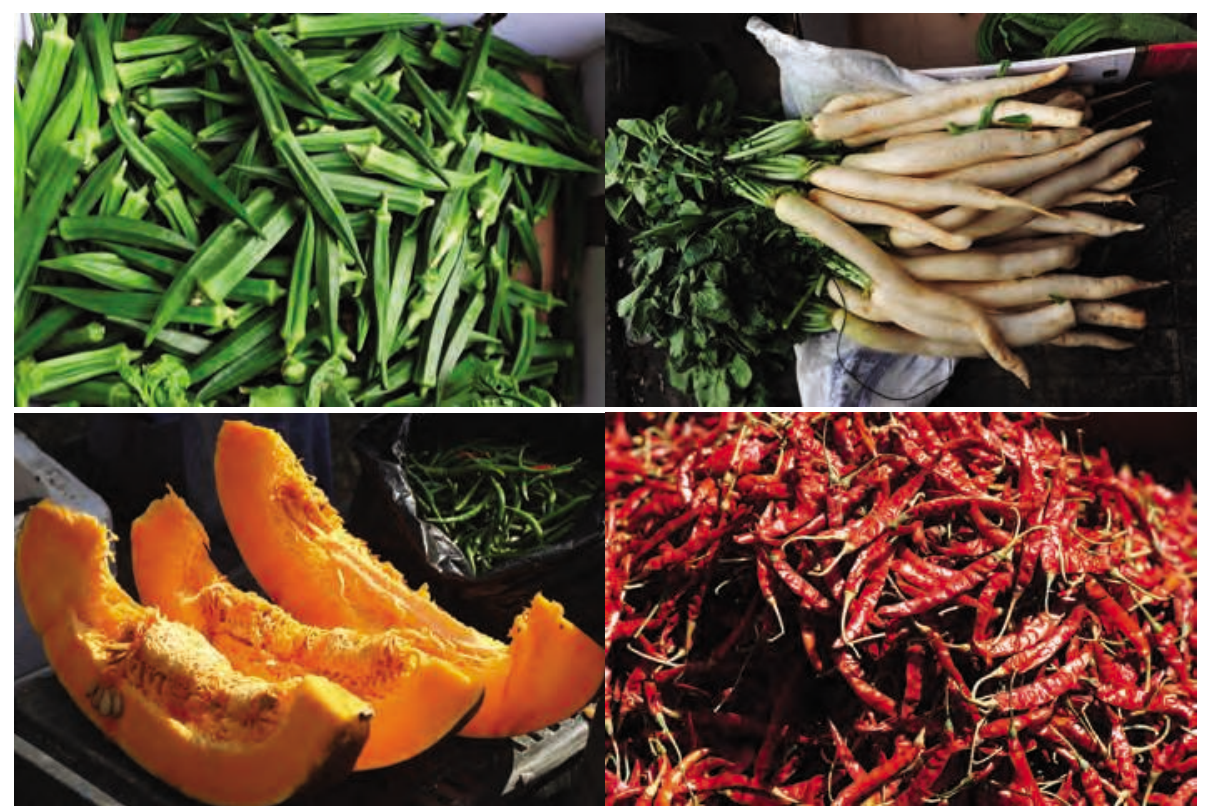

Crédit : H. Kassatly, Marché de Sabra, Beyrouth, 2013 et 2014. 
Dans certains cas, le photographe se doit d'établir des liens avec les migrants à la vitesse éclair et dans l'urgence. C'est le cas lorsqu'il y a besoin de documentation précise comme pour la question des tatouages de fête. Les Bangladais portent sur leur corps deux fois I'an, à I'occasion des fêtes de l'Adha et du Fitr, des tatouages temporaires. L'action photographique est alors tributaire de ce temps précis où le tatouage ne s'est pas encore estompé, il est encore visible, et ce, toujours dans ce moment et cet espace déterminé par le choix du lieu d'enquête : les dimanches du souk. Pour la réussite du projet, il est nécessaire d'obtenir la collaboration des migrants et surtout des migrantes qui portent principalement - même si non exclusivement - ces dessins (Série de photos 8). Elles doivent donc adhérer à la démarche du photographe et ensuite, pour des nécessités d'ordre techniques, se plier à des postures du corps, susceptibles d'assurer la visibilité de ces tatouages par rapport à l'éclairage ce qui signifie pour elles d'arrêter momentanément de courir pour poser.

Et ce, dans un studio virtuel ouvert à tous les regards dans une rue de forte affluence, de bruits assourdissants et de confusion constante. Dans les studios ordinaires, c'est dans une ambiance calfeutrée et privée, à l'abri des regards, que le sujet se livre en quelque sorte à la photographie. À Sabra, c'est dans un lieu public au vu et su de tous et sous les regards étonnés ou narquois des locaux que la migrante répond - ou non - à la sollicitation du photographe avec le risque de ressentir un malaise à voir la relation du photographe au photographié, rendue visible de tous et privé de son corollaire : l'intimité. Ce schéma est d'ailleurs vécu comme incongru et il confond autant les observateurs présents dans le souk, que les migrantes elles-mêmes. II entraîne surtout la stupéfaction de ces dernières de se voir abordées et sollicitées par une personne dont les identités multiples sont toutes sources de surprise. Car il s'agit d'abord d'une photographe, de surcroît libanaise, mais surtout, selon la représentation de la société et en fonction du métier qu'elles exercent, d'une " madame ". Et cette dernière s'intéresse à elles, mais surtout à leur corps, touche leurs mains et se penche vers leurs pieds (qui portent également des tatouages) pour les immortaliser. Mais il ne faut pas perdre de vue que le dimanche est le moment de liberté du migrant, celui où il s'affranchit de sa condition, surtout pour les travailleuses domestiques retrouvant, pour quelques heures, une vie normale. Les femmes expriment cette liberté en venant dans un souk plein de détritus et de mares d'eau stagnante où elles risquent de se salir, vêtues de leurs saris colorés qui tranchent avec la situation délétère du souk (Photos 9 et 10). Et cette dignité retrouvée à travers I'habillement, expression par excellence de soi, leur permet de collaborer parfois sans réserve avec le photographe et de nourrir, de très bonnes grâces, son intérêt pour leur culture. 
Série de photos 8 : Tatouages

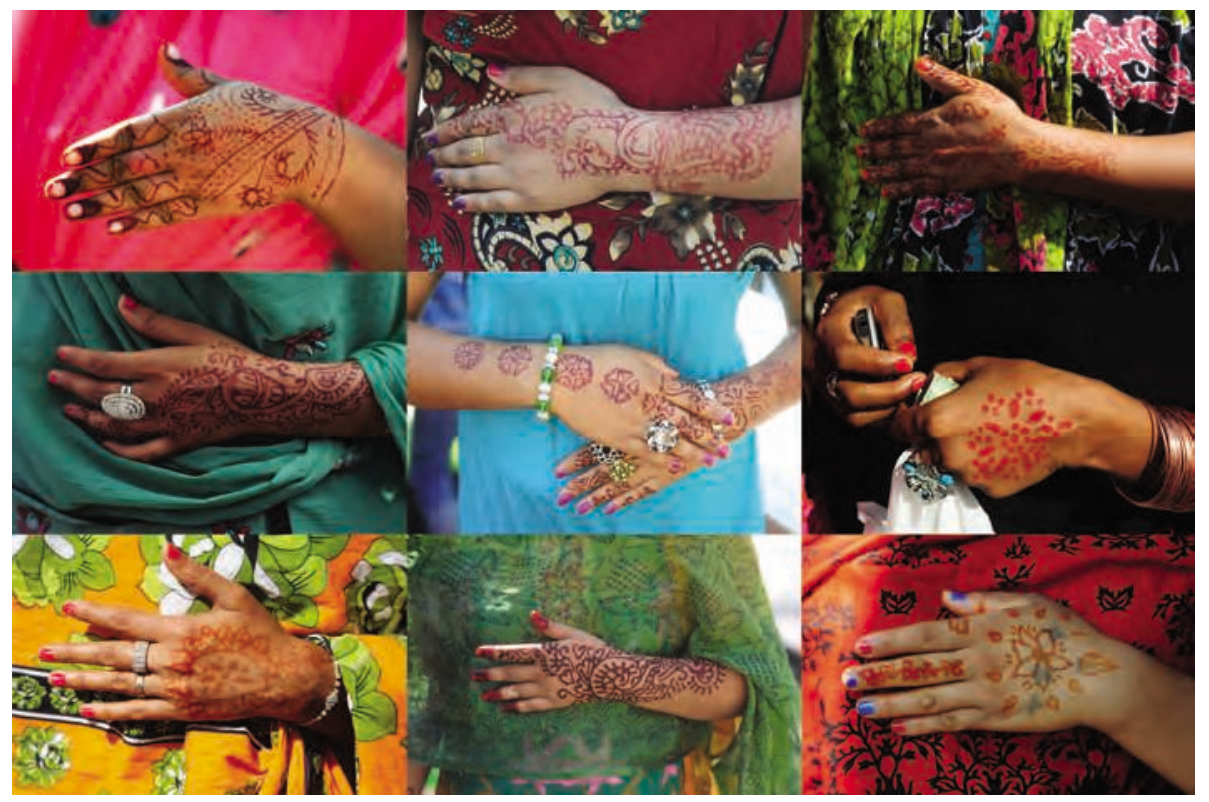

Crédit : H. Kassatly, Marché de Sabra, Beyrouth, 2013.

Photo 9 : La confession intime

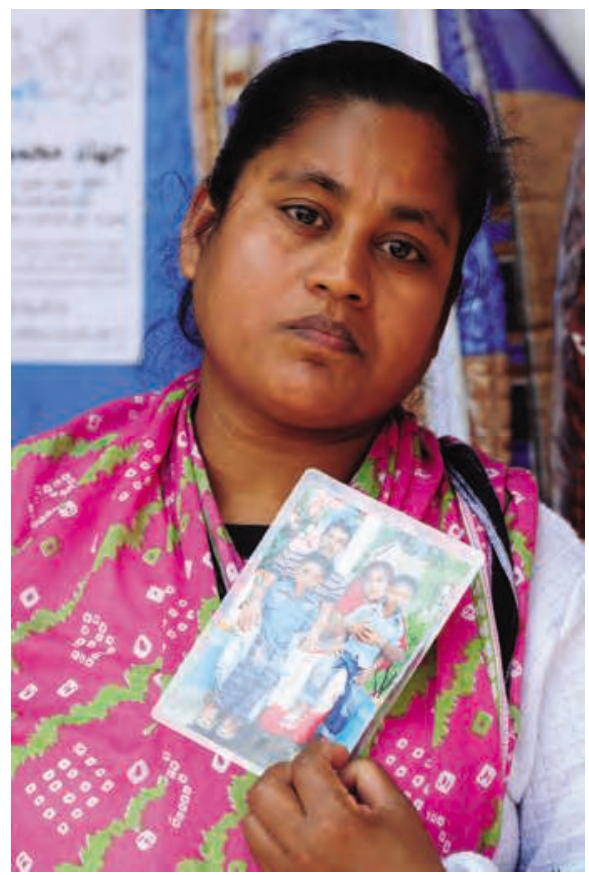

Photo 10 : L'apparat du souk

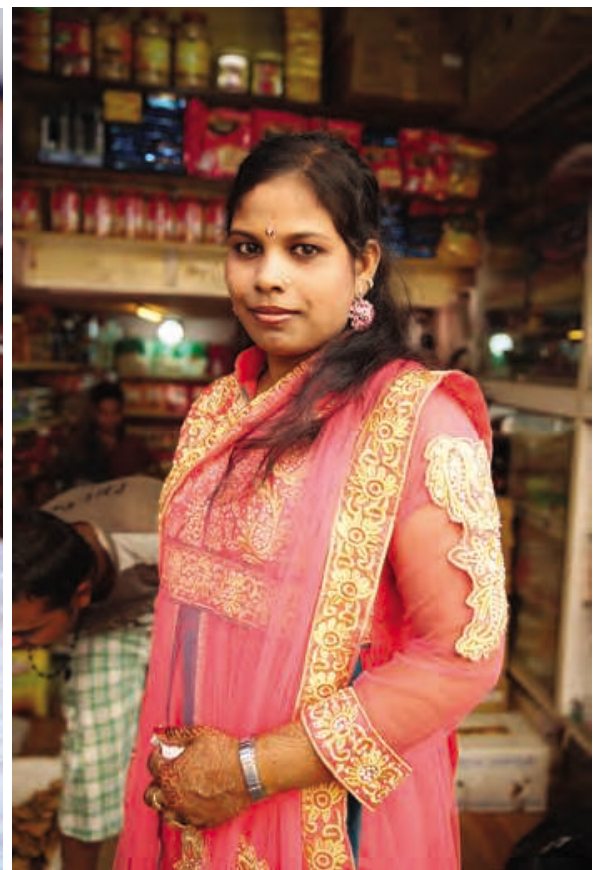

Crédit : H. Kassatly, Marché de Sabra, Beyrouth, 2013 et 2016. 
À l'arrivée, que la présence du photographe oscille entre acceptation et rejet, qu'il doive renégocier sa présence ou pas, l'épreuve du temps et la fidélité au lieu instaurent immanquablement une relation de confiance qui se traduira par cette demande posée tant par les locaux que les migrants : "Photographie-moi s'il te plait " (Photos 11 à 14).

Photos 11, 12, 13 et 14 : La pose en groupe

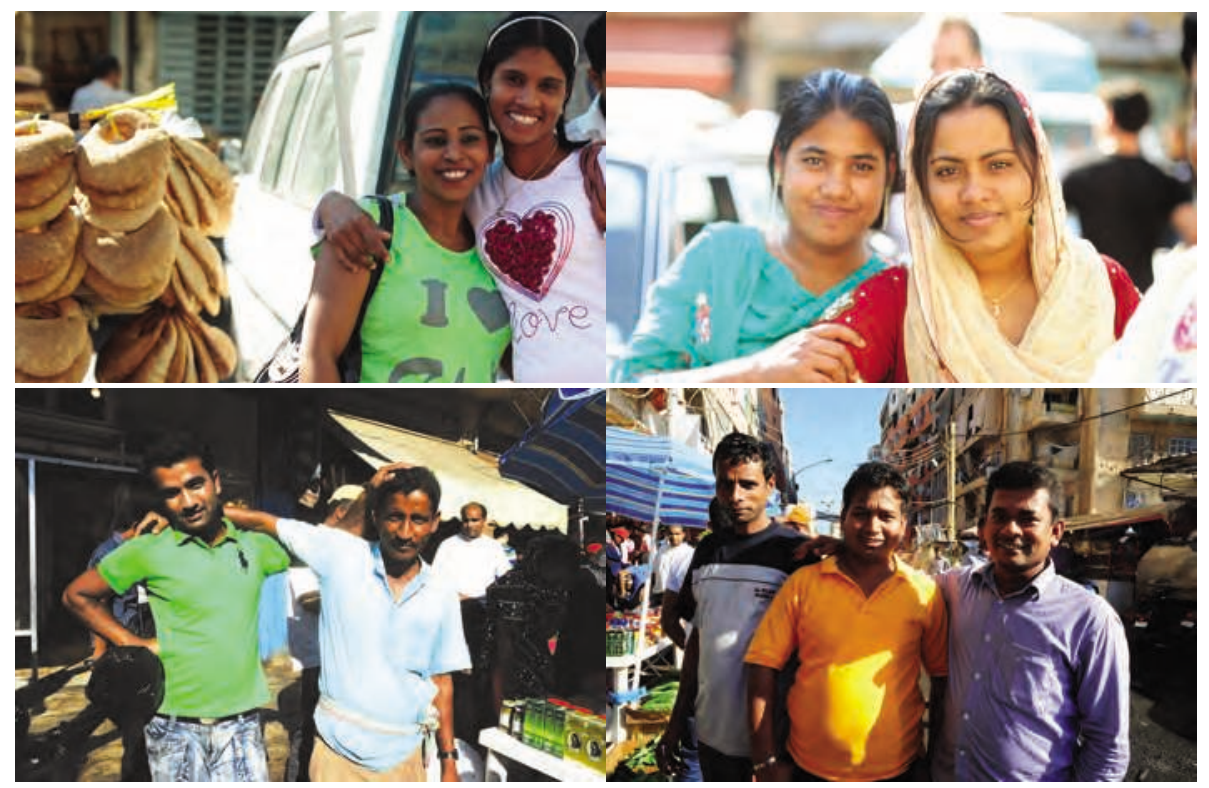

Crédit : H. Kassatly, Marché de Sabra, Beyrouth, 2012, 2013 et 2015.

À l'inverse, ce petit garçon qui ne veut pas être pris en photo instaure une partie de cache-cache avec le photographe qui se répète à chaque rencontre (Photos 15 à 19).

Au vu de cette proximité du photographe avec son terrain, le passeur du souk le sollicite, en public, et accepte de confier son image à cet observateur qui se poste sporadiquement au coin de la rue, consacrant par ce biais, son statut d'habitué (Photos 20 et 21). 


\section{Photos 15, 16, 17, 18 et 19 : Partie de cache-cache avec le photographe}

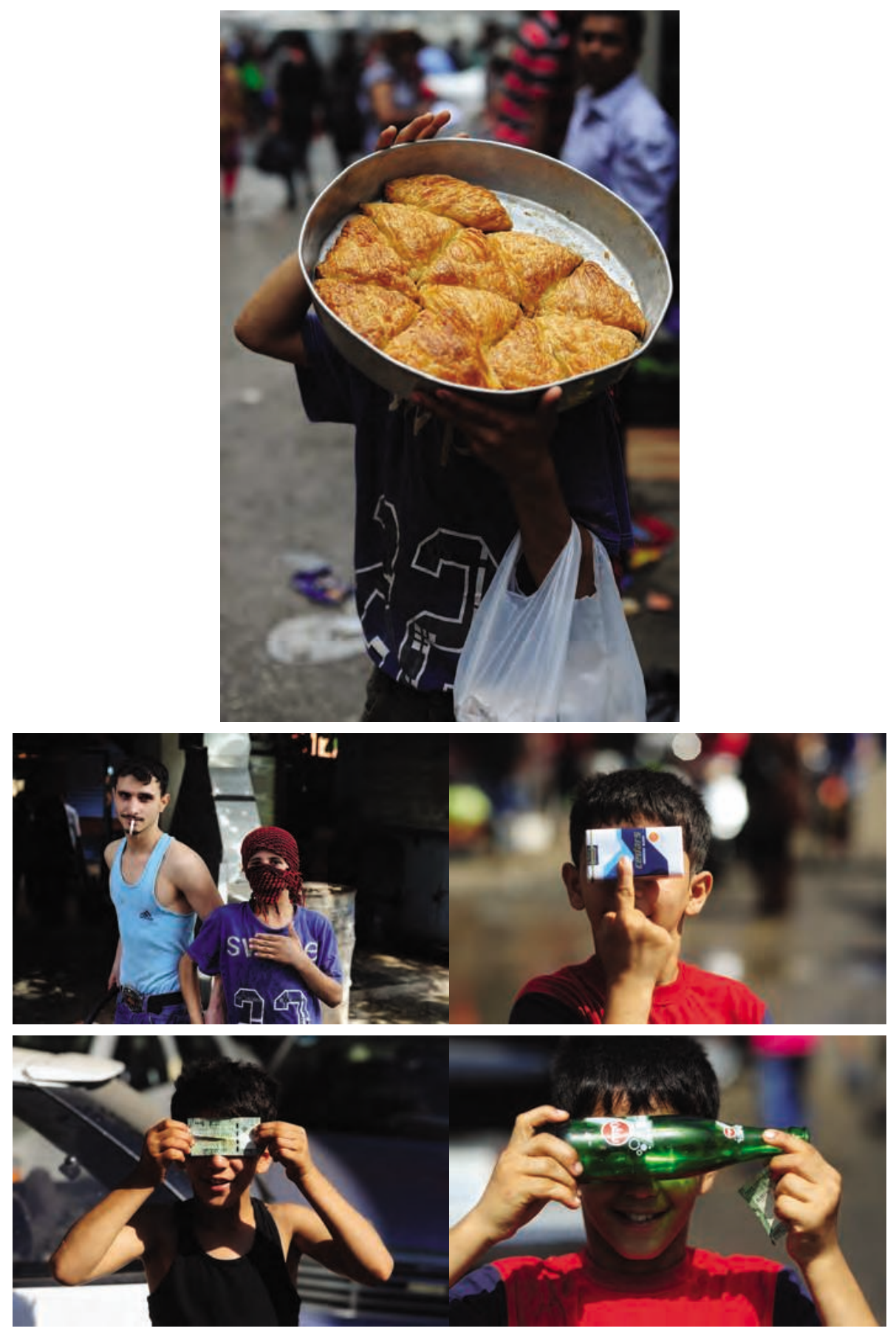

Crédit : H. Kassatly, Marché de Sabra, Beyrouth, 2013 et 2014. 
Photos 20 et 21 : Palestiniens du camp

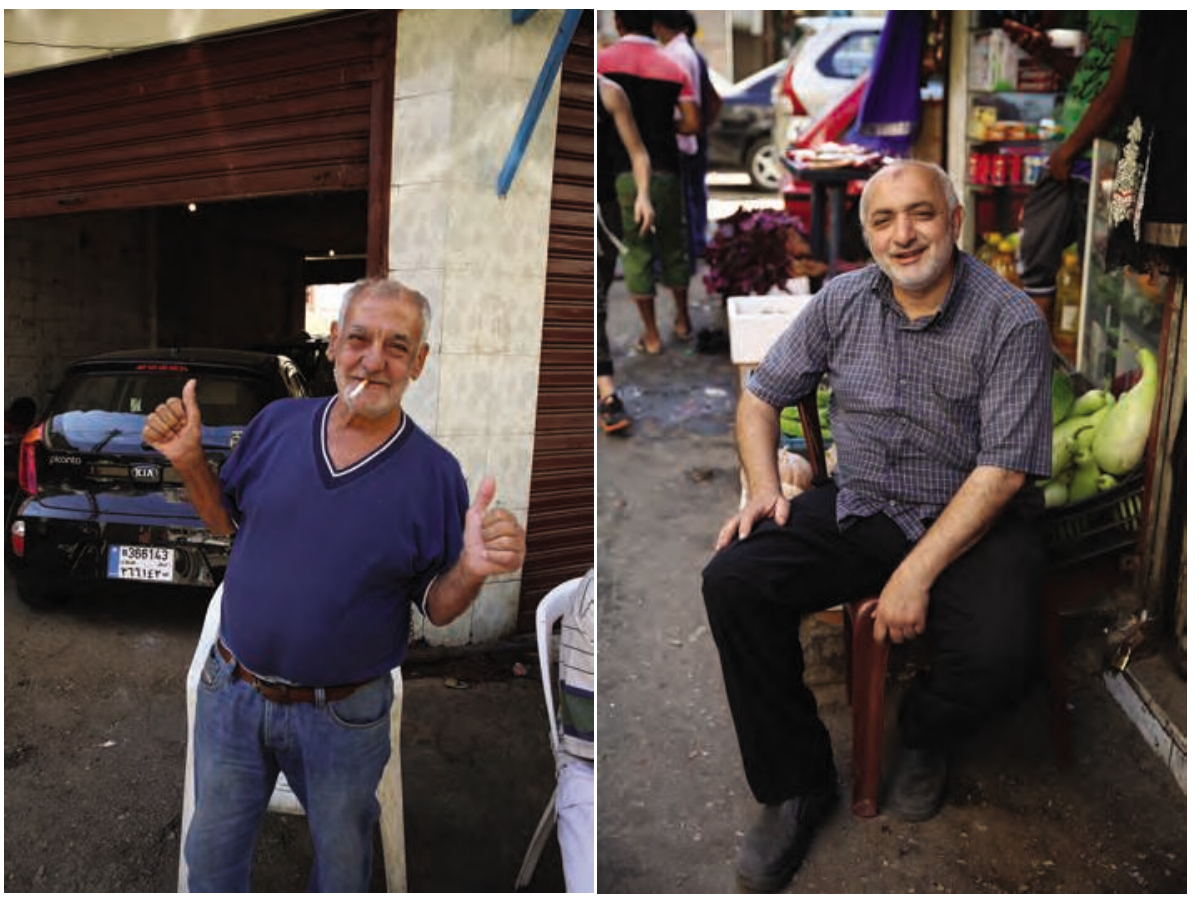

Crédit : H. Kassatly, Marché de Sabra, Beyrouth, 2016.

\section{La vidéo : ethnographies mobiles et observations situées. Contribution de Michel Tabet}

\section{Dispositif filmique}

Le dispositif filmique conçu avec Nicolas $\mathrm{Puig}^{7}$, et mis en œuvre sur le marché de Sabra à Beyrouth, combine deux approches complémentaires : le parcours filmé, qui implique des mouvements à travers la ville, et l'observation située, qui se déroule dans un lieu et un temps donnés. Ces deux démarches reposent sur des prises de vues centrées sur les circulations et interactions de nos enquêtés, elles fonctionnent comme des systèmes d'observation qui relèvent du cinéma direct. Cette posture méthodologique vise à rendre compte de la dimension singulière de la relation à l'espace, de cette pratique de la ville qui varie même quand le cadre des actions et des interactions se duplique et se répète ou qu'il détermine, plus ou moins fortement, les comportements. Ces tournages ont ainsi constitué autant d'explorations immanentes des situations que je filmais. Par exploration, j'entends ici une attitude qui consiste, même quand le tournage s'insère dans le prolongement d'une recherche intensive qui a déjà investi et

7 Qui m'a ensuite " escorté " durant les tournages, à la fois observateur, assistant et intermédiaire auprès de personnes qu'il fréquente régulièrement 
déblayé le terrain selon plusieurs approches différentes et complémentaires, à se remettre dans une attitude de découverte en s'intéressant à ce qui se passe ici et maintenant, quitte à recontextualiser cet ici et ce maintenant au montage ou dans le cadre de visionnages ultérieurs de recherche et d'analyse.

Concernant les parcours filmés, j'ai accompagné des migrants au cours de leurs déplacements dans Beyrouth ${ }^{8}$. J'ai réalisé de cette façon deux ethnographies mobiles, l'une avec Hassan, employé dans une société de nettoyage et vendeur de produits bangladais sur le marché de Sabra, I'autre avec Batcha, chômeur vivant de débrouille et de petits boulots. Le premier habite à Tarik el-Jdidé, un quartier sunnite de la ville, le deuxième à Sabra, dans un ilot majoritairement palestinien ${ }^{9}$. J'ai filmé leurs trajectoires urbaines, par où et comment ils passent, les obstacles qui se dressent sur leurs chemins, la façon dont ils les évitent et les franchissent.

Pour ce qui touche à l'observation située, j'ai réalisé des enregistrements sur deux marchés fréquentés par des migrants. L'un se trouve à Sabra, I'autre à Borj Hammoud, aux périphéries de Beyrouth. Dans ces deux espaces, la caméra a permis de rendre compte des actions et interactions des migrants entre eux et avec les autres populations, libanaises, syriennes, palestiniennes, arméniennes, qui les fréquentent. Elle a aussi aidé à explorer les modalités de partage de ces espaces entre ces groupes, ainsi que les échanges, frictions et ajustements comportementaux et spatiaux que la présence hebdomadaire des migrants bangladais provoque ou induit : transformation des paysages sonores et visuels, vente de nouveaux produits, installation d'étals informels, autant d'éléments qui contribuent à affirmer l'investissement de ces quartiers par les migrants et à en modifier le paysage et l'ambiance.

Dans ces espaces, le film contribue à documenter les transformations de l'environnement à travers l'exploration de deux types d'activités : le commerce et I'expression de soi. Sur la base de ces principes de tournage, il met en évidence la façon dont ces activités participent d'une redéfinition des pratiques et des territoires. Par le commerce, les anciens et les nouveaux, les outsiders, échangent des produits, de l'argent et des marques de curiosité, comme lorsqu'un acheteur libanais s'arrête devant l'étal d'un Bangladais et tente une ethnologie improvisée de ses pratiques alimentaires et de ses goûts culinaires. Par les habits et les productions issues des industries culturelles des pays d'origine, certaines zones de ces marchés deviennent des reproductions d'univers familiers qui transforment ces espaces en marchés ethniques provisoires. En s'installant quelques heures le dimanche matin dans la zone de Sabra, les Bangladais de Beyrouth prennent pied, à la leur façon et dans une zone partagée, au cœur d'un espace communautaire libanais marqué par "la territorialisation des appartenances" (Mermier, 2008 : 10), un processus d'occupation qui passe, pour les populations locales, par l'imposition de symboles (les drapeaux partisans et les portraits des leaders politiques) et des formes d'appropriations qui tournent parfois à la

8 Ces parcours ont fait l'objet d'une convention avec les intéressés donnant lieu à une rémunération pour leur participation à un film universitaire. Le montage final du film leur a été restitué.

9 Le parcours complet de Batsha est disponible sur: https://archive.org/details/Batsha ; celui de Hassan sur : https://archive.org/details/Hassan_201512 
violence. Les images filmées révèlent en partie comment les Bangladais, et les migrants plus généralement, s'y prennent pour s'installer dans ces territoires disputés.

D'une façon plus générale, les deux dispositifs filmiques, le parcours et l'exploration située, sont complémentaires dans la mesure où les personnes dont les trajets ont été filmés participent au marché. Les liens entre le temps du marché et celui du quotidien permettaient de comprendre le fonctionnement du marché comme repère social et urbain. Ces parcours individuels étaient ainsi appréhendés dans la perspective de leur convergence ponctuelle à Sabra.

Pour effectuer ces ethnographies vidéographiques j'ai adopté un système de prise de vue simplifié : une caméra portée à la main munie d'un micro omnidirectionnel venant compléter le micro d'ambiance de la caméra. Ce choix provient d'une volonté de privilégier des tournages d'immersion et d'observation fondés sur un échange entre filmant et filmé. II m'a en outre permis d'avoir une certaine souplesse pour naviguer entre les lignes de partage communautaires et sécuritaires qui maillent la géographie de la ville. II ne s'agit donc pas de prétendre à une observation neutre ou objective de l'expérience des migrants dans Beyrouth, mais de déployer des observations focalisées sur certains aspects de cette expérience tout en assumant les effets et les impacts que l'intrusion du dispositif provoque et induit, comme la méfiance et l'hostilité des autres groupes que nous croisons sur ces lieux où je filme, accompagné de Nicolas Puig, sans couverture et sans autorisation.

Ce choix méthodologique-relationnel s'accompagne aussi d'un choix méthodologique-esthétique: dans la mesure où nous avons voulu saisir des moments particuliers de l'expérience urbaine des migrants et non parler de leur expérience globale de la ville, nous avons mis en œuvre, dans le rendu comme au cours de l'enregistrement, des tournages par fragments. Cette façon de faire provient d'un cheminement propre à mes recherches en anthropologie visuelle de même qu'elle correspond à l'esprit dans lequel j'ai filmé ces parcours et ces situations migrantes.

\section{La question de la séquentialité}

Pour ce qui touche à ma compréhension de l'usage des images en sciences sociales, j'ai montré, en m'appuyant sur des ethnographies filmiques de rituels religieux que j'ai conduites au Liban et en Algérie, que le film ne peut pas restituer la signification ou le déroulement global de l'action rituelle (Tabet, 2014). L'anthropologue qui filme, que ce soit au moment de l'enregistrement comme devant son logiciel de montage, n'a affaire qu'à des bribes ou des séquences de vie "tirées d'une infime partie du cours réel du monde " (Agier, 1996). Son travail consiste alors, comme le souligne Laplantine (2009), à mettre cette réalité en forme plutôt qu'en ordre. Ce privilège accordé à la forme ne renvoie pas au primat du dispositif sur le contenu, mais à celui, manifeste dans le cas du cinéma, qui sert de fondement aux analyses de Laplantine, de l'unicité de la forme et du fond : même si une image ethnographique renvoie à quelque chose d'extérieur, elle reste une image.

Dans le cas d'un film ou d'une séquence, c'est-à-dire d'un dispositif qui travaille principalement sur le temps et sur la durée (Laplantine, 2007), cette 
mise en forme relève d'un travail sur le rythme : à quel moment et pourquoi appuyer sur le bouton d'enregistrement ? À quel moment et pourquoi couper une séquence ? Pourquoi et comment la rapprocher d'une autre ? En poussant cette logique de la fragmentation, nous avons utilisé le film dans le cadre de ce projet comme un moyen de travailler sur des moments, des séquences de la vie. Au lieu de proposer des portraits de migrants, c'est-à-dire des films qui témoigneraient de leur vie dans sa globalité ou de leurs conditions d'existence, nous avons voulu faire des coupes qui permettraient de faire des sortes de prélèvements pour saisir des détails relevant de leur rapport à la ville, à son environnement et à ses autres usagers.

La dimension séquentielle de notre approche à Sabra renvoie aussi au fait que nous effectuons des découpages dans la durée. Cette temporalité n'est pas celle qui oppose la vidéo et le son au caractère instantané de la photographie. Elle renvoie au temps que nous prenons pour chacune des prises, un temps qui n'a rien de systématique, mais qui dure néanmoins le temps qu'il faut pour que nous puissions accompagner un mouvement, une action sonore, jusqu'au bout, au temps qu'il nous faut, en tant qu'observateurs, pour le regarder ou l'écouter. Muni d'une caméra, pris dans le flux de l'action, j'essaie de comprendre ce que je vois, même si souvent cela se fait dans l'urgence. Ce temps prélevé fixe des actions, des interactions et des impressions d'autant plus fluides et contingentes que l'occupation de l'espace par les migrants bangladais relève d'un processus de négociation constante, que leurs positions sur le marché ne sont jamais vraiment assurées.

La dimension séquentielle renvoie enfin à la restitution de cette précarité et de cette fugacité. Nous avons opté pour de courts montages parce que nous $\mathrm{n}^{\prime}$ avions pas l'intention de rendre compte des moindres faits et gestes de ces migrants sur le marché ou au cours de leurs déplacements. Nous avons plutôt cherché à restituer une certaine expérience de la ville, une certaine ambiance, une certaine atmosphère. Les choix de montage procèdent de façon à briser la logique descriptive au profit d'une logique de l'expression dans laquelle j'ai souhaité résumer l'essentiel des situations que nous avons observées : ce sont les visages, les regards, les gestes et les attitudes qui sont mis en avant, comme des réflecteurs de l'environnement. En plus du temps, le cadrage a donc été un élément déterminant de cette explicitation de la relation à l'environnement et de sa signification, à savoir une présence sur le marché qui ne relève pas seulement de l'échange marchand, mais comprend aussi une dimension symbolique.

\section{Les séquences sur le marché}

Les enregistrements sonores réalisés par Nicolas Puig ont fait apparaître la création d'une membrane sonore qui enveloppe à certains moments les locaux présents sur le marché, coupés de leur univers arabe par les voix des Bangladais autour d'eux qui les isolent. L'image vidéo parvient à ce résultat, mais par un autre chemin : selon la position et la valeur de plan que l'on adopte, elle souligne tel ou tel aspect de la réalité, un peu comme l'observateur de Simmel face à une toile : quand il s'éloigne, il a une vue d'ensemble, quand il se rapproche il voit les détails, mais il ne peut jamais saisir les deux en même temps (Simmel, $1981: 88$ ), d'où I'idée simmelienne d'une sociologie multi-perspectiviste particulièrement adaptée lorsqu'il s'agit de concevoir des tournages ethnographiques en mouvement. 
À Sabra, lorsque je filme en plans larges, je vois principalement l'inscription des Bangladais dans le contexte spécifique de ce lieu. En revanche, quand je $\mathrm{m}^{\prime}$ approche et que je serre davantage le plan, j'efface peu à peu ce contexte, qui est dès lors relégué au statut de vague décor. Des hommes et des femmes, originaires d'un même pays, le Bangladesh, occupent alors le champ. Le hors cadre ne disparaît pas, mais sa présence est atténuée. Une image particulière de cet espace émerge ainsi : une image construite ou extraite qui donne à voir un espace qui échappe, ou cherche à échapper à la réalité qui l'entoure, à former une bulle de protection.

C'est peut-être ce théâtre que ces migrants viennent jouer/regarder le dimanche matin à Sabra : en se rapprochant les uns des autres, ils produisent eux-mêmes des représentations et des images dont l'une des principales fonctions seraient d'effacer celles qu'ils perçoivent au quotidien et dont ils sont en quelque sorte exclus. Le rôle de la caméra paraît essentiel en ce qu'elle permet de souligner ce phénomène. Mais pour y parvenir, elle doit pouvoir se rapprocher le plus possible des migrants et le filmant qui le regarde doit s'avancer pour finir par regarder avec eux. Ainsi, en plaçant la caméra à proximité des visages et des épaules, en l'intégrant dans le cercle des échanges inter-migrants, l'image se sature de leur présence et de leurs paroles. Le reste du marché passe hors cadre, même s'il continue de définir le contexte de l'action.

\section{Dispositif de prise de vue des parcours}

S'il existe des possibilités techniques de placer les caméras sur les sujets en gommant la place de l'observateur pour privilégier les données produites par le dispositif d'enregistrement, c'est le cas par exemple pour les parcours sonores effectués dans le cadre de cette recherche, la façon dont j'ai procédé pour les observations filmiques est participative. Elle repose sur une transaction entre filmant et filmé, puis elle conduit l'observateur à marcher dans les pas de l'observé, à se déplacer avec lui, à faire lui-même l'expérience du parcours accompli. II s'agit de regarder la personne en train d'agir, en ajustant la distance pour maintenir une relation du sujet à l'environnement médiatisé par la caméra, en formant une sorte de binôme efficace qui chemine un peu " comme un seul homme ".

Ce dispositif qui s'inspire du cinéma documentaire d'observation, place la caméra à proximité de l'enquêté de façon à ce que ce dernier se sache filmé et qu'il ait conscience des moments où la caméra tourne ou ne tourne pas. Cette perception du dispositif par l'enquêté, dont l'accord préalable est bien sûr nécessaire, l'est aussi par l'ensemble de l'environnement au sein duquel l'observation se déroule : le filmant se retrouve lui-même sous le feu des regards de tous ceux qui, autour de la scène de l'observation observent l'observation. Cette remarque qui semble aller de soi revêt, dans le cas du marché de Sabra, comme dans bien d'autres espaces au Liban, une signification particulière pour deux raisons, I'une structurelle, l'autre conjoncturelle.

La raison structurelle repose sur le fait, souligné par Dahdah (2014 : 39), de la multiplicité des pouvoirs, grands ou petits, qui se partagent le contrôle du marché : pouvoirs des différentes factions politiques, pouvoir des différents corps de métiers (notamment les taxis et les bouchers qui jouissent ici d'une autorité plus importante que les autres commerçants) et enfin pouvoir des petits 
voyous. Chacun de ces groupes affirme sa présence et considère l'intrusion d'une caméra avec méfiance. Des personnes en civil viennent vous demander vos papiers d'identité, d'autres cherchent à saisir votre caméra et il ne faut pas se laisser faire. Pour atténuer ces effets d'interférence, nous avons parfois eu l'occasion de filmer avec des accompagnateurs, en l'occurrence un jeune Palestinien du camp de Chatila. L'interférence s'est alors déplacée des intrusions extérieures vers celles de notre protecteur dont les injonctions et les invectives télescopaient les éléments d'observations que nous enregistrions.

D'un point de vue conjoncturel, l'un des tournages que nous avons effectués a, par exemple, été troublé par le reportage d'une télévision libanaise. Une dizaine de jours avant que nous nous rendions à Sabra, une équipe de journalistes avait fait un sujet sur la qualité de la viande proposée par les bouchers du marché. Ma caméra fut d'emblée perçue comme suspecte et potentiellement hostile. N'ayant pas forcément conscience de ce contexte, j'ai commencé à filmer avant de me faire sévèrement rappeler à l'ordre par l'un des bouchers. Les négociations entre nous ayant échoué, nous nous sommes repliés avec Nicolas Puig autour de l'échoppe à café situé dans un secteur investi par plusieurs commerces pérennes tenus par des Bangladais. Le cafetier, un Palestinien du camp de Chatila, embêté de voir nos travaux universitaires entravés de la sorte, nous recommande de nous placer sous la protection de l'un de ses amis, Salfouh, qui, par hasard, passait par là. Ce dernier, une force de la nature mesurant plus d'un mètre quatre-vingt-dix, nous demande alors de lui indiquer la personne qui s'est mise sur notre chemin. Un peu gênés et pas forcément enthousiastes à l'idée de provoquer une rixe à cause de notre enquête, nous essayons d'esquiver puis finissons par suivre Salfouh jusqu'au carrefour d'où nous venions de nous faire expulser. À peine étions-nous arrivés que le boucher qui nous avait arrêtés s'est levé pour nous renvoyer à nouveau. Mais Salfouh était là et visiblement il était respecté, je tourne alors, plutôt contraint et contrit, quelques images sous sa protection. Nous avons appris par la suite qu'il occupait une place importante dans cette zone et qu'il était redouté pour sa force et son courage dans les rixes.

\section{Les parcours}

Les deux séquences de parcours s'articulent à des questionnements plus larges qui orientent la façon dont nous avons utilisé la caméra de façon à répondre à certaines questions : en quoi un parcours peut-il être considéré ou manifeste-t-il une forme d'appropriation de la ville ? De quelle façon le migrant marche-t-il ? Est-il assuré dans ses pas ? Interagit-il avec les gens qu'il croise ou se fait-il tout petit pour ne pas se faire remarquer? La gamme des possibilités est sans doute infinie et varie en fonction des personnes et des lieux, mais quoi qu'il en soit les séquences que nous avons filmées avec Hassan et Batcha peuvent donc être lues selon deux perspectives :

- La première renvoie aux modalités du déplacement, les techniques du corps et les véhicules que I'on utilise.

- La deuxième à la dimension sociale, c'est-à-dire à la relation à l'environnement et aux autres habitants de la ville parce que le mouvement de la marche, parallèlement aux échanges sur le marché, dessine non seulement les contours d'un rapport à la ville, mais aussi ceux d'un rapport à ses habitants : marcher c'est en quelque sorte s'approprier I'espace (De Certeau, 1980 ; Augoyard, 1979). 
Dans les deux cas que nous avons accompagnés, nous avons eu affaire à des personnes maîtrisant totalement leur espace quotidien, quoique leurs trajets ne relèvent pas des mêmes logiques.

Batcha est à la recherche d'un travail. Particulièrement à l'aise dans l'espace du marché, il n'en sort que très rarement. Ici, il connaît tout le monde et c'est surtout cette forme d'intégration que notre caméra s'est employée à restituer. Ce qui marque dans le trajet de Batcha, c'est à la fois son assurance, il est sur son territoire, et son sens de la communication, qui le conduit à fréquenter les jeunes du quartier. Dans l'entretien que nous avons réalisé avec lui pour commenter son parcours, il nous a assuré que les Palestiniens, c'est-à-dire ses voisins directs, l'aiment bien. Cette appréciation se ressent lorsqu'il marche dans la rue et que les jeunes l'interpellent et prennent de ses nouvelles, surpris de le voir accompagné par une équipe de tournage. Batcha nous assure qu'il est ici chez lui, même si son rêve est de rassembler 5000 dollars pour rentrer au Bangladesh et lancer une affaire.

Le parcours de Hassan est beaucoup plus silencieux que celui de Batcha. La ville grouille autour de lui : il y des bruits, des frictions, des obstacles, mais rien ne l'arrête ou ne le détourne. Hassan est tout entier concentré sur la tâche à accomplir. Nous avons proposé à Hassan de filmer une journée type, même si la notion n'est pas nécessairement pertinente. Cette demande nous a en revanche permis de le suivre sur des lieux familiers : sa maison, son lieu de travail. Tout cela se passe dans la tranquillité de la vie quotidienne. Cette séquence révèle en effet un rapport intégré à la ville : on y a ses habitudes, ses cachettes et ses réseaux.

Hassan se déplace pour aller au travail ou se rendre au marché de Sabra, où lui-même, sa mère et d'autres membres de la famille, tiennent une boutique en semaine et un étal le dimanche. Seule halte dans son parcours, la prière à la mosquée où il s'insère tranquillement parmi les fidèles. À l'opposé de Batcha, Hassan est un entrepreneur. Sa famille tient une boutique toute la semaine dans le quartier de Sabra. Tous les jours après son travail il vient donner un coup de main. Le dimanche, il s'installe à quelques mètres de là, à un carrefour plus fréquenté, afin de vendre encore davantage. II y a chez lui une recherche de maximisation de l'investissement à travers un positionnement multiple dans la ville. Le parcours filmé fonctionne ici comme une mise en relief de la dimension fonctionnaliste et utilitariste du rapport de Hassan aux lieux qu'il traverse et dont il fait usage.

Nous avons aussi eu la chance de filmer Hassan au travail, car il est employé dans une société qui assure des services de nettoyage à l'ambassade de France. Nous n'avons donc pas eu besoin d'avoir l'accord de l'employeur, mais seulement celui de l'ambassade. II faut dire qu'il n'est pas facile de filmer les migrants au travail : il y a non seulement la question de la légalité de ce travail, il y a aussi celle des conditions de travail. À l'ambassade, les prestataires sont tous déclarés, ils portent une combinaison et s'occupent principalement du nettoyage. 


\section{La fluidité et les accidents de parcours}

Hassan et Batcha se déplacent donc d'un pas assuré. Ils connaissent bien les parcours qu'ils empruntent et ne semblent freinés par aucune sorte de méfiance. Les incidents auxquels Hassan a été confrontés au cours de notre tournage ont été induits par la présence de notre caméra : en plusieurs occasions nous avons été interrompus dans notre parcours.

La première fois, c'était en sortant de la maison de Hassan, où un voisin, étonné de l'intérêt que des chercheurs français pouvaient porter à un travailleur bangladais, a tenu à nous souligner, avec les relents racistes et condescendants que cela suppose, le peu d'estime qu'il avait pour ces voisins sales qui occupaient sans doute leur taudis de façon illégale. Selon lui, la présence des Bangladais faisait perdre de la valeur au quartier. Plus loin, à proximité du siège de la sécurité intérieure dans le quartier du musée, des policiers en civil nous arrêtaient, car nous étions en train de filmer dans une zone sécurisée. En fin de journée, alors que nous nous rendions au marché de Sabra, c'est un jeune homme qui a tenté de se saisir de la caméra, considérée par lui comme un objet menaçant. À chaque fois, nous avons dû gérer nous-mêmes ces accrochages tandis que Hassan, selon une stratégie soulignée par Dahdah (2014), se mettait en retrait et adoptait une attitude d'évitement.

Ce faisant, nous pouvons considérer que cette tendance à se mettre à l'écart, révélée par l'intrusion d'un dispositif qui a bouleversé l'ordre habituel du déplacement, fait partie intégrante du rapport de ces migrants à la ville : en temps normal, et même si cela se passe de façon plus discrète, l'assurance du déplacement cache probablement une tactique globale de l'évitement. Pour avancer, Hassan passe sans doute son temps à éviter toute une série d'obstacles, physiques comme sociaux qui se dressent devant lui. Ce jour-là, la caméra en a révélé quelques-uns.

Nous avons été frappés par la fluidité des déplacements de nos enquêtés, constat confirmé à chaque fois que nous avons fait face à un obstacle : nos informateurs disparaissent sans laisser de trace, ils poursuivent leur chemin, rien ne semble les arrêter. Mais la fluidité n'implique pas l'absence d'obstacles, elle $s^{\prime} y$ ajuste. II y en a sans doute beaucoup plus que ce que nous avons pu voir, mais ce que nous avons constaté, c'est la façon dont les deux migrants que nous avons accompagnés tentent de glisser sur ces difficultés pour continuer à avancer.

\section{L'approche sonore. Contribution de Nicolas Puig}

L'étude sonore complète la description visuelle photographique et filmique de l'environnement humain à Sabra et apporte une perspective inédite sur le social en privilégiant l'ouïe sur les autres sens temporairement suspendus. II $s^{\prime}$ agit d' " aborder l'homme et le monde à partir de la dimension sonore " dans un contexte scientifique où la matière visible et le regard sont privilégiés dans les enquêtes sociologiques et anthropologiques. L'étude des " modes audibles" offre ainsi " la possibilité concrète d'aborder autrement les phénomènes sociaux" (Augoyard, $2003: 26)$. 
Le dispositif sonore que j'ai mis en place à Sabra a pour objectif de saisir l'environnement sensible par le son, d'approcher les perceptions et de transcrire les relations au moyen de différentes techniques d'enregistrement (micro fixe et utilisation du micro stéréophonique binaural).

Les enregistrements proviennent de micros fixes disposés aux endroits du marché caractérisés par leur forte densité humaine. De nombreux enregistrements ont été effectués depuis la charrette du marchand de bananes syrien Abu-I-Izz qui constitue un poste d'observation intéressant. Une partie des vendeurs bangladais s'installait d'ailleurs sur cette pointe avancée du marché faisant face à l'avenue de Sabra avant de refluer vers une aile du marché située une dizaine de mètres plus loin. J'ai également effectué des enregistrements en m'équipant du micro binaural (deux micros de petite taille qui s'insèrent dans les oreilles comme des écouteurs intra-auriculaires, reliés à un enregistreur numérique) et en me tenant dans des endroits divers. Enfin, une dernière technique consiste à demander à des informateurs d'effectuer un parcours sonore qu'ils commentent ensuite.

L'approche sonore ouvre trois perspectives, chacune étant construite à partir d'un mode d'observation dominant. La construction discursive de l'étranger transparaît des données fournies par les parcours sonores, les relevés d'interactions isolent de petites scènes sociables entre migrants et installés, l'environnement sonore connaît des modifications importantes lors de la présence des Bangladais.

\section{La construction discursive de l'étranger à travers le parcours sonore}

Le parcours sonore constitue la première méthode déployée sur le marché de Sabra ${ }^{10}$. Avec Hala Abou Zaki ${ }^{11}$, nous avons demandé à deux habitants du camp de réfugiés palestiniens de Chatila d'effectuer un parcours entre le camp et le marché voisin de Sabra. Nous avons équipé ces deux personnes d'un micro binaural. Le dispositif d'enregistrement permet ainsi la restitution exacte et spatialisée des sons qui arrivent aux oreilles de la personne qui enregistre, une sorte de 3D sonore. Nos informateurs ont ensuite été invités à réécouter au casque en notre compagnie leur trajet enregistré et de nous décrire l'enregistrement de leur trajet (description que nous avons donc aussi enregistrée).

Nous avons cherché à amener les participants et leurs proches à prendre position sur le peuplement et la démographie de la zone dans le cadre d'une discussion collective. II s'agissait d'aborder le thème de la présence considérée comme importante des migrants asiatiques, notamment du Bangladesh, sur le marché de Sabra, et de repérer les différents stéréotypes en circulation au moyen d'une méthode adaptée. Cette démarche était nécessaire pour apporter quelques éléments sur la construction discursive d'autrui, une sorte de cartographie humaine perçue du marché. Elle a en outre apporté des éléments de compréhension de la différenciation sonore entre le camp et la zone commerciale de Sabra, première étape de notre exploration du marché à travers son imbrication avec les quartiers environnants.

10 Sur cette méthode, voir Battesti et Puig (2016).

11 Doctorante à I'EHESS, membre du programme CEDRE. 
Cet exercice permet de susciter une réflexivité sur la présence des étrangers à partir d'une expérience sonore qui se traduit ici par l'audition de différentes langues et accents arabes (palestiniens, libanais et différents accents syriens) le long du parcours. Les deux informateurs notent de façon récurrente la présence $\mathrm{d}$ 'accents arabes et de langues étrangères. En ce sens, les parcours mettent en avant la dimension de Babel horizontale que revêt à présent le marché de Sabra.

Le premier parcours concerne une femme syrienne d'une trentaine d'années, Um Abdallah, qui réside à Chatila depuis son mariage en 1994 avec un Palestinien du Liban né en 1971, boucher à Sabra, qui est présent au moment du commentaire. Um Abdallah raconte avoir connu un certain ostracisme, confinant au racisme parfois, de la part de sa belle-famille et des habitants du camp, du fait de sa nationalité syrienne. Elle a dû également faire face aux accusations visant le régime syrien qu'elle était supposée personnifier : son mari originaire du quartier de Sabra s'est réfugié en Syrie avec sa famille durant les années 1980, fuyant l'invasion israélienne et les violences qui l'ont accompagnée et est rentré au Liban au début des années 1990 avec son épouse syrienne.

Le second parcours ${ }^{12}$ est effectué par Jamal, un homme né à Chatila en 1965 dont la famille fait partie des premiers arrivants au camp peu après son exil de Jaffa vers le Liban en 1948. Jamal a dû s'éloigner du camp durant quelques années lorsque les factions pro-syriennes de Chatila étaient trop menaçantes (il est militant du Fatah). II est ensuite rentré dans son camp dont il commente les évolutions démographiques et urbaines avec beaucoup de nostalgie.

Deux modes relationnels paraissent pouvoir être dégagés à partir des parcours et des discussions qu'ils suscitent. Le premier mode est celui de l'évitement et le second celui de la spécification. Ces deux modes sont univoques : ils ne s'établissent pas dans le cadre de relations en direct avec les étrangers, mais dans celui d'un rapport distancié. Ce sont donc des points de vue du dehors ou des points de vue éloignés qui ne s'appuient pas sur des échanges réguliers avec les migrants, mais témoignent d'une fréquentation passive. Le mari de notre informatrice syrienne, Abu Abdallah, est en revanche en contact avec les migrants qui viennent parfois s'approvisionner dans la boucherie où il travaille.

Jamal vit un sentiment de déclassement et de perte de repères par rapport à la transformation de l'espace à Chatila. Dans son analyse de la situation de Sabra, il dénonce de façon récurrente la dégradation de cet espace qui représente pour lui et ceux de sa génération une référence identitaire et politique forte.

Jamal affirme ne pas être en contact avec les migrants du Bangladesh dans son quotidien, même s'il les connaît. II distingue ainsi les vendeurs syriens, libanais et palestiniens et les Bangladais « qui viennent à des jours déterminés, le dimanche, qui ont leurs magasins et leurs produits précis qu'ils vendent ici " (Commentaire du parcours de Jamal, 7 min $28 \mathrm{~s}$ ). Ces derniers sont désignés ensuite comme les "vendeurs étrangers" (14 $\min 14 \mathrm{~s})$.

12 Pour accéder aux transcriptions intégrales des commentaires des deux parcours suivre http://vbat.org/spip.php?article749. Les transcriptions de l'arabe oral à I'arabe écrit ont été réalisées par Manal Jaafar - entrer [parcours sonores de Jamal] dans la fenêtre de recherche web et suivre le lien. 
Jamal reconnait confondre les Asiatiques du marché, pour lui ce sont tous les mêmes et il ne sait pas voir qui est du Bangladesh, d'Inde ou du Sri Lanka, mais il sait que des Bangladais viennent à présent s'installer dans le camp.

Les voix des acteurs arabes du marché sont distinguées dans le parcours (voix syriennes, libanaises et palestiniennes), y compris dans le cas de Palestiniens s'exprimant avec un accent libanais (12 min $18 \mathrm{~s}$ ), mais la connaissance préalable de la personne joue dans le cas précis un rôle important dans l'identification.

En revanche, Jamal n'a pas la même précision pour décrire les voix des migrants asiatiques puisqu'il qualifie de Sri-Lankais un groupe de Bangladais en train de discuter (18 min 35 s). Peut-être utilise-t-il le gentilé " Sri-Lankais " comme un terme générique pour désigner les migrants asiatiques au Liban. Cet usage idiomatique s'est répandu au début des années 1990 avec l'arrivée massive des employées domestiques asiatiques. Une réplique donnée dans la pièce de théâtre Le Jardin de Sanayeh, mise en scène par de Roger Assaf, en rend compte avec malice : "Srilankatî srilankiyyeh" (ma Sri-Lankaise est sri-lankaise).

Les premières personnes qu'il rencontre au sortir de sa maison lors de son parcours sont des enfants syriens turbulents. II confie qu'il les aurait volontiers réprimandés s'ils avaient été palestiniens. Pour Jamal, "être du camp " ( $m i n$ al-mukhayam), c'est être palestinien, il ne dira pas d'une personne qu'elle " est du camp ", si elle ne fit qu'y résider simplement (20 min $1 \mathrm{~s}$ ).

Aussi semble-t-il glisser sur ce monde des migrants et du marché et n'avoir que peu de prise sur lui. Abu Abdallah, le mari d'Um Abdallah qui a effectué le second parcours sonore, développe quant à lui une vision assez circonstanciée des nationalités en présence.

Il distingue ainsi les étrangers « arabes » -Syriens, Irakiens, Libanais, Palestiniens des migrants asiatiques - les Philippins, les Sri-Lankais et les Bangladais - qui occupent le marché la nuit du samedi jusqu'au dimanche matin (38 min $41 \mathrm{~s}$ ).

À l'instar de Jamal et comme fréquemment sur le marché, Abu Abdallah emploie souvent le nom du pays pour désigner les ressortissants, Philippines, Sri Lanka ou bien Bangladesh pour finalement les regrouper selon leurs habitudes culturelles et religieuses : tous mangent du piment, écoutent tous la même musique, la seule différence entre ces groupes nationaux relèverait de leur appartenance religieuse (musulmans, chrétiens et sikhs).

"Bangladesh et Sri Lanka, ceux-là se retrouvent surtout le dimanche, la nuit du samedi au dimanche et le dimanche matin" (38 min $41 \mathrm{~s}$ ).

"Au Liban, on trouve maintenant le monde entier : Sri Lanka, Bangladesh, Égyptiens, Philippins. Toute l'Asie presque s'y retrouve [...]" (41 min $21 \mathrm{~s}$ ).

Son épouse Um Abdallah débute son parcours par un achat à Sabra où elle capte dans ses micros des échanges entre une femme libanaise et deux Sri-Lankaises. Après que la première a assuré aux migrantes qu'il n'y avait pas de tsunami au Liban, elles continuent de discuter de ce sujet. Um Abdallah rapporte l'échange : "Elles parlent de tsunami. Elle lui a dit: "Et s'il y avait un tsunami au Liban [la migrante] ?." Alors elle lui répond [la Libanaise] : "On meurt tous, normal" ". 
Ensuite, la Sri-Lankaise échange avec son amie en cingalais (18 min $35 \mathrm{~s}$ ). Elle entend également les voix locales qu'elle différencie aisément : "La plupart sont palestiniennes et libanaises, quelques-unes syriennes " (15 min $33 \mathrm{~s})$.

Puis, elle pénètre dans le marché aux légumes de Sabra pour faire un achat. Dans son commentaire, elle décrit la foule autour d'elle, foule qu'elle éprouve à présent par le son. Dans le flux sonore, les voix des vendeurs syriens très présents dans cette partie du souk émergent ( $29 \min 33 \mathrm{~s}$ à $30 \mathrm{~min} 30 \mathrm{~s}$ ).

Enfin, I'environnement sonore du camp est suffisamment spécifique pour que Um Abdallah se localise grâce aux voix des enfants qui jouent dehors la nuit tombée, ce qui signale l'entrée dans un espace déjà en partie privatisé, un prolongement de l'espace domestique ( 1 h 5 min et $6 \mathrm{~s}$ ).

Cet effet de seuil est également noté par Jamal, qui identifie au changement d'ambiance sonore le passage du camp à l'avenue de Sabra : "Ici, je suis arrivé au début du camp qui donne sur l'avenue Sabra parce qu'il y a beaucoup de bruit, de deux-roues et de voitures " ( $3 \min 13 \mathrm{~s}$ ). Ces effets de seuils pointés par les informateurs montrent l'importance de la sensorialité, ici l'audition, dans l'établissement des familiarités avec I'environnement. Ils tracent les frontières sensorielles entre l'ordinaire du camp et la zone plus ouverte et mobile de Sabra où se produit en priorité la rencontre avec les étrangers proches et lointains.

\section{Formules du contact}

Des échanges de personnes à personnes, des adresses à l'égard des clients étrangers du marché, émergent de la matière sonore prélevée sur marché. Isolés sous la forme de relevés d'interaction, ces échanges esquissent quelques "formules du contact " entre les installés et les migrants. On se situe dans le domaine de la communication interpersonnelle qui désigne " toute situation où des individus identifiés [ici des installés et des Bangladais] et en nombre limité entrent dans un processus d'échange d'information signifiante et intentionnelle » (Augoyard, $2003: 30)^{13}$.

Ces formules représentent les atomes d'un univers cosmopolite ${ }^{14}$ émergeant $^{\prime}$ de la coprésence, confirmant ou infirmant les nombreux gestes de cadrage

13 II s'agit de propos publics échangés au vu et sus de tous sur le marché. La captation sonore dans ce cas ne fait que suppléer à la mémoire du chercheur en restituant les échanges dans leur intégralité, y compris les silences, hésitations, accents et façon de dire, de grande valeur pour I'analyse. II revient au chercheur dans son parcours sonore de distinguer les conversations publiques des échanges privés et d'adapter en conséquence sa prise de son.

14 Cosmopolitisme réfère ici davantage aux ajustements pragmatiques effectués dans une coprésence problématique qu'à une notion de tolérance et de bienveillance à l'égard de l'autre. Cette vision renvoie à la cosmopolitique que Millot (2008: 52) définie ainsi : " le cosmopolitique renvoie non pas à une conception pacifiée d'un universel abstrait, mais prend acte du fait que le monde commun n'est pas donné, déjà là, reconnaissable, mais toujours d'une certaine manière à construire ensemble, dans la complexité, la diversité et le conflit ". On évoquera donc des moments particuliers où émergent des " situations cosmopolites " qui à l'échelle de la ville " redessinent les territoires urbains pris dans le jeu de la mondialisation et des mobilités individuelles et collectives ". Ces situations n'abolissent pas les frontières, elles les déplacent : " elles rejettent la clôture, mais n'en contribuent pas moins pour autant au marquage contextualisé de frontières symboliques " (Hily et Rinaudo, 2003 : 55). 
affirmant la hiérarchie des installés sur les outsiders. Le cadre relationnel global issu du rapport social structurel qui ordonne hiérarchiquement les positions de chacun se dissémine en un grand nombre de cadres possibles " jamais ou ouverts ou fermés, mais progressivement ouverts ou progressivement fermés " (Laplantine, $2005: 81$ ).

Certaines formules sont récurrentes. Elles concernent l'échange autour des produits, les questions d'hospitalité et I'homogénéisation des étrangers.

Un échange enregistré fournit un exemple de reconnaissance de l'autre établie autour d'un produit : le concombre amer (la margose, Momordica charantia L.). II s'agit d'un légume très apprécié des habitants du sous-continent indien (Inde, Sri Lanka et Bangladesh), encore inconnu au Liban. C'est un légume relativement cher et difficile d'usage, mais cette caractéristique permet de passer du temps à en expliquer les spécificités et la façon de le préparer (cf. Encadré 1).

\section{Encadré 1 : Korrola (2'22) $)^{15}$}

mp3.320kbps.30juin2013

(microbinaural)

(extrait)

URL : https://soundcloud.com/sabra-sonore/corollamp3320kbps

Discussion entre un Bangladais et un Libanais. Un autre homme du Bangladesh et le marchand interviennent ponctuellement. La discussion a lieu devant les cartons de légumes asiatiques à même le sol disposés devant les bouchers locaux sur le carrefour de Sabra. On entend continuellement des hommes et des femmes converser en bengali, quelques bribes d'échanges en arabe. Le bruit des hachoirs à viande et les klaxons complètent l'environnement sonore de l'interaction.

- (Homme libanais) Shou haydha? Ma ba'ref. Shou haydha?

"C'est quoi ce truc, je ne sais pas, c'est quoi ?"

- (Homme bangladais 1) Haydha min bangladesh haydha.

"Ça, ça vient du Bangladesh. "

- Eyh, btitubkhu ya'ni?

"Oui, vous le cuisinez c'est-à-dire ?"

- Boss, haydha murr.

"Regarde, c'est amer. "

- Eyh?

"Oui ?"

- Haydha murr minshan biya'mal ma'u batata bazella bess kwayes

[...] jisem kwayes [...] bazella bya'melu byakul kwayes. Bess yiju bangladesh hadha biyakhdu.

"C'est amer. On le fait avec des pommes de terre et des petits pois, mais c'est bon. C'est bon pour le corps. Avec des petits pois, ils le font, c'est bon. Simplement les Bangladais [ils viennent du Bangladesh] viennent et ils en prennent " [...].

15 Ces extraits sonores référencés par leur titre sont en ligne sur les pages Sabra Sonore du site SoundCloud. 
Ce dialogue forme une situation de "négociations identitaires marchandes " selon les termes de Ma Mung (2006:2) qui les définit ainsi : " dans les transactions marchandes mettant en cause des partenaires spécifiés par leur appartenance à des groupes, se négocient, par le truchement des biens matériels échangés, des valeurs et des signes d'appartenance ". Ici, les biens sont dépositaires d'une partie de l'univers sensoriel du migrant et constituent la base de l'échange. La transaction ouvre un espace de neutralité. Elle offre un cadre où les hiérarchies sont abolies le temps d'un échange sur le produit, elle permet ainsi de créer des familiarités entre les personnes qui peuvent se répercuter à l'échelle des groupes en présence.

De nombreuses formules concernent les formes variables de l'ascendant que s'autorise l'installé sur l'outsider migrant. Ces formes s'étalonnent depuis les plaisanteries échangées avec les étrangers jusqu'à des comportements beaucoup plus humiliants qui prennent la forme d'incessantes provocations de la part d'adolescents (imitation stigmatisante de la langue des migrants, gestuelles déshonorantes, etc.).

Joseph (1997 : 137) considérait ce type de comportement comme un " envers de l'hospitalité " figurant une "épreuve majeure de l'étrangéité ". Celle-ci résulte du : " droit que s'accorde l'autochtone à ne rien cacher de sa curiosité, de sa fascination ou de sa répulsion, la jouissance sans entraves du regard inquisiteur ". Cette réflexion lui fut suggérée par la vision d'un groupe d'hommes rigolards entourant des baigneurs noirs sur une plage en ex-Yougoslavie. Cet envers de l'hospitalité se traduit par les libertés que s'accordent certains locaux, les moins établis d'entre eux, vis-à-vis des migrants.

Une autre série de formules concerne l'uniformisation des étrangers. Ainsi de I'usage du gentilé (nom du pays) pour désigner les ressortissants du Bangladesh est fréquente et ne prend pas nécessairement une connotation négative. On s'adresse ainsi souvent à eux en les qualifiant du nom de leur pays, pour leur demander de "dégager " (zih ya Bangladesh), mais on peut également entendre des adresses bien plus civiles comme "ya habibi ya Bangladesh " (mon cher Bangladais). On peut aussi tenter de leur vendre des produits, comme ce poissonnier palestinien qui interpelle les Bangladais pour leur proposer des sardines (ilish) (cf. Encadré 2).

\section{Encadré 2 : Bangladesh (0’58)}

mp3.320 kbps.09juin2013.microbinaural

URL : https://soundcloud.com/sabra-sonore/bangladeshmp3320-kbps

Marqueurs sonores : hachoir à viande, klaxons

Cris : Karaz karaz karaz.

"Cerises [x 3]. "

Poissonnier palestinien : Ilish (x 6).

Klaxons, conversations entre des migrants.

Autre poissonnier palestinien: Hadha taza, al-yum.

"C'est frais, du jour."

Poissonnier palestinien : Bangladesh, Bangladesh Bangladesh,

Bangladesh, ilish ilish ilish ilish.

Voix en bengali, moteur scooter.

Poissonnier palestinien : Ilish ilish, bangladesh (x 4). 
Le poissonnier palestinien appelle d'éventuels clients du Bangladesh en criant le nom de leur pays pour vendre ses sardines locales. II les désigne par le nom vernaculaire bengali d'une espèce de la famille des sardines (Clupeidae), le ilish (Tenualosa ilisha), poisson côtier et d'estuaire que l'on retrouve au Bangladesh et en Asie du Sud. Le ilish est considéré comme un mets de premier choix dans tout le sous-continent indien et il est même le poisson national du Bangladesh. La tactique commerciale du marchand palestinien témoigne d'une bonne connaissance d'éléments de la culture nationale de ce pays. Les transitions sémantiques (langues et produits) des majoritaires vers les minoritaires sont nombreuses et nuancent la logique asymétrique du marché en montrant une certaine transitivité des échanges matériels et symboliques.

Certains installés emploient dans une interaction avec un Bangladais I'adresse plus sympathique, mais non moins homogénéisante de sadíq (copain). Dans l'enregistrement " sadîq ", le fils d'Abul-I-Izz apostrophe depuis la charrette de son père les Bangladais sur le marché en les appelant "sadîq ". Tous les migrants se retournent, ne sachant auquel d'entre eux s'adresse l'adolescent. Cela amuse beaucoup Abu-I-Izz et son fils. Un migrant intervient au bout d'un moment pour lui demander de cesser (cf. Encadré 3).

Encadré 3 : Sadîq (copain) (0’52)

320kbps.7juillet2013.microbinaural
URL : https://soundcloud.com/sabra-sonore/sadîq052
Voix en bengali, récitation coranique en provenance de la mosquée.
Le fils d'Abu-I-Izz (Abdel-Razzaq) hèle les migrants bangladais en
les appelant par leur surnom usuel : " sadîq ", jusqu'à ce que I'un
d'entre eux le reprenne, ce qui amuse Abu-I-Izz.
- (Abdel-Razzaq) : Sadîq (...), sadîq, (...) sadîq, (...) entaaa sadîq (toi
là, copain), le Bangladais se retourne : Enta sadîq! (tu es un copain).
(...) Sadîqa (copine) sadîq (copain).
- (migrant) : Il proteste en s'adressant au fils d'Abu-I-Izz " ça suffit
avec ton sadîq, là ! "..

\section{Membrane sonore}

Enfin, la présence des Bangladais et, dans une moindre mesure, celle d'autres migrants venus de différents pays d'Asie et d'Afrique modifient les propriétés sensorielles du marché de Sabra le dimanche. Dans les zones du marché où ils se concentrent, la nappe sonore formée par les discussions publiques à haute voix des nombreux Bangladais entre eux voile les marqueurs sonores locaux - voix arabes, bruit des hachoirs à viande, klaxons et moteurs de voiture et de deux roues, cliquetis des tasses de café entre les doigts des vendeurs ambulants - et modifie sensiblement l'environnement habituel.

Une première écoute des enregistrements montre toute la richesse sonore du marché, les subtiles variations d'intensité de rythme et de fréquences qui en marquent les seuils et signalent les lieux de densités relationnelles. Dans cet environnement, les Bangladais rassemblés en divers endroits célèbrent ensemble le plaisir de se retrouver entre compatriotes et de converser dans leur propre langue. Le public est mixte, hommes et femmes, et plutôt jeunes. Une joyeuse insouciance émerge de rassemblements éphémères où les Bengalais s'échangent les dernières nouvelles, s'enquièrent de la situation des uns et des 
autres et déploient éventuellement quelques arguments de séduction.

Ces conversations, paroles et appels des marchands et clients bangladais qui se pressent en certains lieux du marché, forment une membrane sonore qui sépare les marchands locaux du reste de l'environnement plus spécifiquement arabe du marché. Cette membrane plonge l'auditeur dans un univers sensoriel inédit, elle modifie le paysage sonore du carrefour de Sabra. Elle constitue un fond sonore d'une intensité moyenne qui s'établit dans la durée.

Dans les enregistrements "Membrane.sonore.1 " et "Membrane.sonore.2 ", l'entremêlement des voix des Bangladais est traversé par les bruits de la circulation des véhicules sur le carrefour à une heure de grande affluence. Cette immersion entretient pour les installés à cet endroit du marché la sensation de ne plus avoir prise sur leur monde, d'en être dépossédé par les étrangers (cf. Encadré 4).

\section{Encadré 4 : Membrane.sonore.1 (2'52)}

Membrane.sonore.1 (2'52)
320kpbs.Sabra 01.09.2013.microbinaural
URL : https://soundcloud.com/sabra-sonore/mur-sonore-micro-fixe-252
Au plus fort du marché bangladais, en fin de matinée le dimanche,
les voix des Bangladais qui se tiennent nombreux devant la
charrette du marchand syrien Abul-Izz, sont prépondérantes et
forment une membrane sonore qui l'isole de son environnement
arabe. Les voix portent peu et restent de volume modéré
néanmoins. Différents éléments sonores traversent la membrane
comme le son des hachoirs à viande des bouchers, les klaxons,
les moteurs de véhicule, les appels et les cris des locaux qui
sont d'un volume bien supérieur. On entend à plusieurs reprises
le son des tasses de café qui s'entrechoquent (0'09 à 0'13 par
exemple), une conversation en arabe en arrière-plan est perceptible.
L'enregistrement traduit assez fidèlement les sons auxquels Abu-l-
Izz, lui-même assez silencieux, est exposé.

Les membranes sonores se déplacent suivant les migrants. En mars 2015, j'enregistre un groupe important de marchands et de chalands bangladais concentrés sur le bord de l'avenue Sabra, juste après le carrefour (cf. Encadré 5).

Encadré 5 : Membrane.sonore.2 (2'03)

320kpbs.Sabra 29.03.2015.microbinaural

URL : https://soundcloud.com/sabra-sonore/mursonore2

Nombreux klaxons (nous sommes au bord du carrefour Sabra).

Malgré I'intense circulation, on entend distinctement les conversations des Bangladais qui forment un mur sonore qui sépare les boutiques du reste de l'environnement. 1'15: voix arabe (" Arib arib, ma tsakkar attarik, arib» (" Avance, avance, ne bloque pas la route, avance "). Je stationne devant une boutique de couettes, coussins et couvertures entre la boutique et les Bangladais installés le long de la route. Le mur sonore est dense, mais le marchand tolère la situation, car une partie de ses clients du dimanche matin sont précisément des migrants. Tandis que les Bangladais stationnent et discutent, on entend parfois des bribes de conversations de passants arabes. 
Les enregistrements montrent les variations de l'intensité sonore sur le marché, dont la membrane sonore, qui se déploie durant de longues minutes avant de rétracter pour complètement disparaître vers $14 \mathrm{~h}$. La vie perceptive s'accorde à la présence sonore. Elle est faite d'oscillations entre des temps d'attention, d'engagement, et d'autres de déprise, des " moments lents".

Presque rien ne se passe dans ces moments lents, la membrane est temporairement occultée par un réaménagement sensoriel et cognitif : on n'y prête plus attention. Chacun est à sa rêverie, en discussion avec lui-même, arrangeant ses cageots du bout du pied ou jouant avec son smartphone.

Puis on se reconnecte sur l'environnement : Abu-I-Izz focalise tout à coup son attention sur les voix étrangères qui l'entoure et se gausse de leur sonorité. Devant lui, à la vue d'un épais rideau de pluie qui progresse au loin, Hassan, le vendeur bangladais s'empresse de recouvrir sa marchandise d'une bâche de plastique anticipant un déluge à venir.

Les acteurs du marché sont sensibles aux variations de l'atmosphère, eux qui travaillent en extérieur, soumis aux quatre vents. Alors que nous sommes tous les deux silencieux depuis un peu de temps, par une matinée chaude et ensoleillée, Abu-I-Izz me signale : "Tu as vu depuis quelques secondes, il y a une petite brise ". Je fais alors la remarque qu'il doit se féliciter de vivre dans un appartement aéré, lui qui réside au septième étage d'un immeuble de Chatila. II me répond que "C'est vrai, il y a de l'air ; par contre, il n'y a pas d'ascenseur ".

\section{Conclusion}

Ce texte écrit à trois mains est nécessairement inachevé, comme les sont les petites histoires qui se tissent dans cette marge urbaine de Beyrouth. II se veut avant tout méthodologique. Son objet est de détailler les différents dispositifs audiovisuels déployés sur un lieu de densité et d'hétérogénéité humaines, un marché cosmopolite de Beyrouth, et d'en montrer l'intérêt pour les études migratoires : I'intrusion de la photographie, les ethnographies mobiles, les séquences de la vie collective et leur dimension sonore. II est encore trop tôt pour en tirer des conclusions définitives, l'enquête se poursuit et le matériel d'ores et déjà récolté $\mathrm{n}^{\prime} \mathrm{a}$ été que très partiellement exploité.

L'analyse s'élabore progressivement à partir de séquences visuelles et de fragments sonores, agencés selon différentes lignes de cohérence. Elle multiplie les entrées problématiques et les accroches de connaissance. Les dispositifs mis en place éclairent différentes variations sociales et sensibles prenant place sur le marché qui dessinent un univers relationnel instable.

Nous avons tracé ici quelques-unes de ces lignes qui apparaissent en filigrane des descriptions méthodologiques. Elles concernent bien entendu le rapport à l'autre, mais aussi la perception de soi, les schèmes corporels, la souveraineté sur son corps, les tactiques de l'évitement et celles de la communication.

Des variations qui nous incitent à ne pas durcir outre mesure dans l'analyse de la forme de la relation entre migrants et installés pour faire émerger les hésitations et les inventions communicationnelles, comme elles attirent notre 
attention sur la circulation discontinue des significations et l'alternance des moments lents et des séquences d'engagements. L'enquête de Sabra montre l'existence de longues plages de la coprésence sans échange de face-à-face, des temps où chacun est dans son monde. Laplantine (2005: 80) insiste : " il n'y a que des bribes de sens qui sont comme vous et moi : elles apparaissent, elles disparaissent, elles bougent sans cesse et sont loin d'être présentes à tous moments".

La relation et le sens circulent de façon discontinue, alternant moments de classement d'autrui, reconnaissance de ses propriétés, écoute, divagation des pensées et focalisation sur les sensations du moment. Le parcours sonore, où les rencontres sociables alternent avec une attention flottante pour différents marqueurs sonores (moteur, voix d'enfants, cris lointains, klaxons), témoigne particulièrement bien de cela.

Pour les acteurs du marché, les fonctions indicielle et informationnelle de la vision et de l'ouïe médiatisent la relation à l'environnement, sous-tendent des interprétations et alimentent les représentations. Ces deux aspects sont étroitement articulés, car la perception sensorielle et la représentation sont dans un dialogue continu.

La reconnaissance de la différence est un des supports de la rencontre. Par exemple, l'étrangeté est domestiquée par la médiation des produits. Tandis que les différentes manifestations d'hospitalité soulignent, nuancent ou renforcent la situation asymétrique comme dans le cas de l'envers de l'hospitalité, manifestation de la préséance de l'installé sur l'outsider. L'uniformisation des migrants dénommés "copains " (sadîq) ou Bangladesh, du nom de leur pays, est une solution pragmatique élaborée par les installés pour circonscrire ces " autres" étonnants.

À ce stade, on mesure l'importance de Sabra en tant qu'espace d'expérimentations sociales où des acteurs immergés dans un environnement dont ils sont aussi les producteurs imaginent des formules sociales dont il s'agirait d'apprécier ensuite les disséminations depuis cette marge vers d'autres secteurs de la société libanaise. 


\section{Références bibliographiques}

Agier Michel (1996) Les savoirs urbains de l'anthropologie, Enquête, 4, [en ligne] consulté le 11/05/2016. URL : http://enquete.revues.org/683

Augoyard Jean-François (2003) Une sociabilité à entendre, Espaces et sociétés, Ambiances et espaces sonores, 115, pp. 25-42.

Augoyard Jean-François (1979) Pas à pas, Essai sur le cheminement quotidien en milieu urbain, Paris, Seuil, 185 p.

Battesti Vincent and Puig Nicolas (2016) "The Sound of the Society". A Method for Investigating Sound Perception in Cairo, The Senses and Society, 11 (3), pp. 298-319.

Dahdah Assaf (2014) Négocier sa place chez l'autre. Les migrants dans les espaces palestiniens à Beyrouth, Revue Européenne des Migrations Internationales, 2 (30), pp. 25-47.

De Certeau Michel (1980) L'invention du quotidien, Paris Gallimard, 380 p.

Hily Marie-Antoinette et Berthomière William (2006) Décrire les migrations internationales, Les expériences de la co-présence, Revue Européenne des Migrations Internationales, 22 (2), pp. 67-82.

Hily Marie-Antoinette et Rinaudo Christian (2003) Cosmopolitisme et altérité. Les nouveaux migrants dans I'économie informelle, Tsanta, 8, pp. 48-57.

Jarrigeon Anne (2015) Sociologie visuelle et droit à l'image. La demande d'anonymat en question, L'Année sociologique, 1 (65), pp. 225-246.

Joseph Isaac (1997) L’Hospitalité : Prises, réserves, épreuves, Communications, 65, pp. 131-142.

Lallemand Emmanuelle (2010) La ville marchande : enquête à Barbès, Paris, Téraèdre, $190 \mathrm{p}$.

Laplantine François (2009) Son, images et langage; anthropologie, esthétique et subversion, Paris, Beauchesne, $202 \mathrm{p}$.

Laplantine François (2007) Penser en images, Ethnologie française, 37 (1), pp. 47-56.

Laplantine François (2005) Le social et le sensible : introduction à une anthropologie modale, Paris, Téraèdre, $220 \mathrm{p}$.

Ma Mung Emmanuel (2006) Négociations identitaires marchandes, Revue Européenne des Migrations Internationales, 22 (2), pp. 83-93.

Mermier Frank (2008) Liminaire, in Frank Mermier Dir., Liban : espaces partagés et pratiques de rencontre, Beyrouth, Presses de I'Ifpo, pp. 9-13.

Meyer Michaël (2010) Tu veux ma photo ? Droit de regard et droit à l'image dans la sociologie visuelle, in Sylvain Laurens et Frédéric Neyrat Dirs., Enquêter: de quel droit ? Menace sur l'enquête en sciences sociales, Vulaines-sur-Seine, Éditions du Croquant, pp. 155-186.

Millot Virginie (2013) Ambiance pluraliste et socialisation urbaine : ethnographie de I'espace public du quartier de la Goutte d'Or à Paris, $H A L$, [en ligne]. URL : http://hal.archives-ouvertes.fr/hal-00833997 
Millot Virginie (2013) Pluralist ambiance and urban socialisation: Ethnography of the public space in the Goutte d'Or neighbourhood of Paris, [trad. de Neil O'Brien], Ambiances, [online]. URL: http://ambiances.revues.org/223

Millot Virginie (2008) Cosmopolitique de I'espace public, Culture et recherche, 114-115, pp. 52-54.

Noussia Antonia and Lyons Michal (2009) Inhabiting spaces of liminality: Migrants in Omonia, Athens, Journal of Ethnic and Migration Studies, 4, pp. 601-624.

Raulin Anne (2009) Minorités urbaines : des mutations conceptuelles en anthropologie, Revue Européenne des Migrations Internationales, 25 (3), pp. 33-51.

Simmel Georg (1981) Sociologie et épistémologie, Paris, Presses Universitaires de France, $240 \mathrm{p}$.

Tabet Michel (2014) Ethnographie filmique des rituels religieux : ce qu'on observe, ce qu'on filme, ThéoRèmes, 7, [en ligne] consulté le 11/05/2016. URL : http://theoremes.revues.org/687 


\section{Houda Kassatly, Nicolas Puig et Michel Tabet}

\section{Le marché de Sabra à Beyrouth par l'image et le son. Retour sur une enquête intensive}

Ce texte a pour ambition d'apporter une contribution méthodologique aux études sur les formes de coprésence dans les lieux de densité et d'hétérogénéité humaines. Il décrit les dispositifs audio-visuels déployés sur un marché de Beyrouth situé dans le quartier de Sabra, leur réception par les acteurs et les effets de connaissance attendus. Tous les dimanches, des outsiders migrants asiatiques principalement originaires du Bangladesh insèrent leurs propres activités sociables et commerciales sur ce marché occupé par des installés arabes (Palestiniens, Syriens et Libanais). L'objectif est d'approcher l'expérience sensorielle et relationnelle des acteurs en présence à partir de séquences de diverses natures, photographiques, filmiques et sonores. Sabra représente un espace social où des acteurs immergés dans un environnement sensoriel, dont ils sont aussi les producteurs, imaginent les formules communicationnelles de la coprésence.

\section{Sabra Market in Beirut through Pictures, Videos and Sounds: Methodological Perspectives}

This paper aims to provide a methodological contribution to study relationship in the dense and heterogeneous human places of gathering. It describes the audio-visual method used in a Beirut market located in the district of Sabra, near Chatila. Every Sunday, Asian migrants mainly from Bangladesh spread out their own sociable and commercial activities in the market usually occupied by Palestinians, Syrians and Lebanese. The research seeks to describe how the outsiders takes place in this place and to analyze the sensorial and relational experiences of the actors involved in the market from recorded sequences of various kinds: pictures, videos and sounds. Sabra is a social space where, actors, outsiders and dominants, immersed in a sensorial environment they are also producing, imagine different ways to communicate together.

\section{El mercado de Sabra de Beirut a través de la imagen y el sonido. Resultados de una encuesta en profundidad}

Este artículo pretende ser una contribución metodológica para aquellos estudios que tratan las formas de co-presencia en lugares de densidad y heterogeneidad humanas. En él se describen los dispositivos audiovisuales desplegados en un mercado de Beirut situado en el barrio de Sabra, cómo los actores reaccionan antes ellos y los efectos de conocimiento esperados. Todos los domingos, los outsiders migrantes asiáticos, originarios principalmente de Bangladesh, integran sus propias actividades sociales y comerciales en este mercado, ocupado por árabes (palestinos, sirios y libaneses) anteriormente instalados. El objetivo es acercarse a la experiencia sensorial y relacional de los actores presentes a partir de secuencias de naturaleza diversa: fotográficas, fílmicas y sonoras. Sabra representa un espacio social donde los actores, inmersos en un ambiente sensorial producido por ellos mismos, imaginan las fórmulas comunicacionales de la co-presencia. 